\title{
Diagnosis for Covariance Structure Models by Analyzing the Path
}

\author{
Ke-Hai Yuan, Chrystyna D. Kouros, and Ken Kelley \\ University of Notre Dame
}

\begin{abstract}
When a covariance structure model is misspecified, parameter estimates will be affected. It is important to know which estimates are systematically affected and which are not. The approach of analyzing the path is both intuitive and informative for such a purpose. Different from path analysis, analyzing the path uses path tracing and elementary numerical analysis to identify affected parameters when a 1-way or 2-way arrow in a path diagram is omitted. It not only characterizes how a misspecification affects model parameters but also facilitates a good understanding of the relation among different parts of the model. This article introduces and studies this technique and, for commonly used models, provides detailed analysis to identify the directions of change for various model parameters. Examples based on real data show that the technique of analyzing the path can reliably predict the direction of change in parameter estimates even when the true model is unknown. Conditions that interfere with the results are also discussed and advice is provided for its proper application.
\end{abstract}

Structural equation modeling (SEM) plays an important role in understanding the relations among multivariate data (Bollen, 1989; MacCallum \& Austin, 2000). In a typical application of SEM, one has a substantively justified model, which is quite likely unacceptable when statistically tested. The model modification index (Sörbom, 1989), Lagrange multiplier (LM) test (Chou \& Bentler, 1990), or

Correspondence should be addressed to Ke-Hai Yuan, Department of Psychology, University of Notre Dame, Notre Dame, IN 46556. E-mail: kyuan@nd.edu 
automated specification search procedures (Marcoulides \& Drezner, 2001, 2003) can be used to locate paths ${ }^{1}$ that may contribute to substantial improvement in model fit. ${ }^{2}$ However, model modification cannot guarantee that the resulting model will reproduce the population. Of course, if a causal relationship exists, a valuable model has to reflect the actual relationship among latent and manifest variables. Such an issue involves both the substantive knowledge of the data and statistical tools for model evaluation. In this paper, we will develop a tool to study parameter biases in a substantially justified but misspecified model. A model will be regarded as correctly specified if it reproduces the population covariance matrix when the parameters are at a set of numbers called the population values.

Although a substantive model may barely fit the data, the model can still be accepted based on a few of many fit indexes in standard software. After accepting a model, the parameter estimates will be used for description or inference. For example, researchers interested in a mediation model obtain evidence about the strong mediation effect by testing whether the direct effect (a parameter estimate) is significantly different from zero (Baron \& Kenny, 1986; Cole \& Maxwell, 2003). Similarly, in the standardized solution, error variance estimates may be used to describe the reliabilities of the measurements. However, when a model is misspecified, parameter estimates and their derived statistics may be biased. Although for a given data set it might be difficult to find a better model that is closer to the data, as measured by a discrepancy function, and theoretically justified we still need to understand the effect of model misspecification, especially the possible biases a misspecification might bring. Only after a good understanding of the possible biases in the model can we apply the SEM methodology more scientifically and reach valid conclusions. In the applied literature many parameter estimates from grossly misspecified models are unduly elaborated, not because better models do not exist but because the SEM literature lacks effective techniques for fully understanding the consequence of a misspecified model. The goal of this article is to provide intuitive and valuable tools for model diagnosis so that SEM can be a better research methodology.

The effect of model misspecification has been studied by many authors (Bentler \& Chou, 1993; Bollen \& Ting, 2000; Cole \& Maxwell, 2003; Cragg, 1968; Farley \& Reddy, 1987; Gallini, 1983; Hausman, 1978; Luijben, Boomsma, \& Molenaar, 1988; Raykov \& Penev, 2002; Saris, Satorra, \& Sörbom, 1987;

\footnotetext{
${ }^{1}$ We call any one-way or two-way arrow in a path diagram a path. A path represents either a fixed or a free parameter in the model. A nonzero path corresponds to a nonzero value of the parameter.

${ }^{2}$ Saris, Satorra, and Sörbom (1987), Luijben and Boomsma (1988), and Kaplan (1990) suggested that the model modification index or LM test should be used in conjunction with expected parameter change (EPC) or standardized EPC. Bentler (1990) and Bollen (1990) provided alternative views about EPC/standardized EPC.
} 
Silvia \& MacCallum, 1988; Sörbom, 1975; Yuan \& Bentler, 2004). For a covariance structural model $\mathbf{M}(\boldsymbol{\theta})$, Yuan, Marshall, and Bentler (2003) observed that the set of parameter estimates $\hat{\boldsymbol{\theta}}$ is a continuous function of the sample covariance matrix $\mathbf{S}$ defined through an estimating equation. Let this function be $\hat{\boldsymbol{\theta}}=\mathbf{g}(\mathbf{S})$. As the sample size $N$ increases, $\mathbf{S}$ and $\hat{\boldsymbol{\theta}}$ converge in probability to their population values $\boldsymbol{\Sigma}$ and $\boldsymbol{\theta}_{*}$, respectively; consequently $\boldsymbol{\theta}_{*}=\mathbf{g}(\boldsymbol{\Sigma})$. The model is correctly specified if $\boldsymbol{\Sigma}=\mathbf{M}(\boldsymbol{\theta})$ for certain $\boldsymbol{\theta}$, denote it as $\boldsymbol{\theta}_{0}$, then $\boldsymbol{\theta}_{0}=\mathbf{g}\left(\boldsymbol{\Sigma}_{0}\right)$, where $\boldsymbol{\Sigma}_{0}=\mathbf{M}\left(\boldsymbol{\theta}_{0}\right)$. Thus, the population value of $\hat{\boldsymbol{\theta}}$ corresponding to a correctly specified model is $\boldsymbol{\theta}_{0}$ and to a misspecified model is $\boldsymbol{\theta}_{*}$. The same model can be misspecified when $\boldsymbol{\Sigma}$ is different from $\boldsymbol{\Sigma}_{0}$. Let $\theta=g(\boldsymbol{\Sigma})$ be a component of $\mathbf{g}$. When $\boldsymbol{\Sigma}$ is close to $\boldsymbol{\Sigma}_{0}$, by applying the mean value theorem in calculus, we have

$$
\Delta \theta=\theta_{*}-\theta_{0} \approx \sum_{i j} a_{i j}\left(\sigma_{i j}-\sigma_{i j 0}\right),
$$

where $a_{i j}$ is the partial derivative of $g$ with respect to the $i j$ th element of $\boldsymbol{\Sigma}=\left(\sigma_{i j}\right)$. Equation 1 implies that when the $i j$ th element of $\boldsymbol{\Sigma}$ is perturbed or slightly changed, the magnitude and sign of $a_{i j}$ decide how $\theta_{*}$ is going to differ from $\theta_{0}$. Let the bias in a parameter estimate $\hat{\theta}$ be defined as $\theta_{*}-\theta_{0}$. When $a_{i j}>0$, a positive perturbation on $\sigma_{i j}$ will cause a positive bias in $\hat{\theta}$; when $a_{i j}=0$, changes in $\sigma_{i j}$ will have no effect on $\theta_{*}$ or lead to a zero bias in $\hat{\theta}$. The formula in Equation 1 is rigorous but does not permit a thorough understanding of the relation of all the model parameters. Yuan et al. (2003) also briefly introduced the idea of analyzing the path, which provides similar information as Equation 1 but enables better comprehension of the relation of parameters within a model. Unfortunately, the authors only studied a single case when an error covariance is excluded from a confirmatory factor model. This article thoroughly studies the approach of analyzing the path and extends it to other types of models as well as to excluding paths other than error covariances. We also show that analyzing the path can equally apply when variables are excluded from a model. We hope that, unlike Equation 1, the technique developed here will be accessible to psychometricians as well as applied researchers.

Analyzing the path to identify the effect of model misspecification on parameter estimates is different from path analysis, where certain rules are necessary to identify causal effects among a set of related variables (see Boker \& McArdle, 2005; Li, 1975; Loehlin, 2004; Wright, 1920). Analyzing the path studies how parameters change in response to a misspecification in the model by applying elementary numerical analysis to model-implied covariances. It only requires a basic knowledge of covariance algebra or path tracing (see Bollen, 1989, pp. 21-22; Loehlin, 2004, pp. 26-27). When a nonzero path between two variables is excluded, the model is not adequate in explaining the covariances 
of the corresponding indicators. Existing paths connecting the manifest or latent variables need to explain this ignored covariance. Typically, the shortest path connecting the two responds strongly. Other paths may also be affected due to their sharing parameters. Suppose a path with a positive loading between $x_{1}$ and $x_{2}$ is ignored and the model-implied covariance by the existing path is $\theta_{1} \theta_{2} \theta_{3}$. Then, to properly explain $\operatorname{Cov}\left(x_{1}, x_{2}\right)$, at least one of $\theta_{1}, \theta_{2}$, and $\theta_{3}$ has to be greater than its population value corresponding to the correctly specified model. Typically, the parameters $\theta_{1}, \theta_{2}$, and $\theta_{3}$ will also appear in the model-implied variances and covariances of the other variables in the model. In such a case, it is possible that one of $\theta_{1}, \theta_{2}$, and $\theta_{3}$ becomes much greater, whereas the other two become slightly greater or even smaller to adjust the other parts of the model. In short, if a direct path between two variables is excluded, the other paths in the model indirectly connecting these two variables will need to "make up" for the ignored covariance, resulting in potential biases in parameter estimates of other paths in the model. The technique of analyzing the path is useful in determining how the different paths in the model may be affected.

\section{Example 1}

The data set of Wheaton, Muthén, Alwin, and Summers (1977) contains two indicators of alienation, anomie, and powerlessness, which were measured at both 1967 and 1971. The measured background variables are education and occupational status index. This data set has been used as an example in SEM software manuals and textbooks (e.g., Bentler, 1995; Bollen, 1989; Jöreskog \& Sörbom, 1993). The full model for the six variables as presented in the literature is represented by Figure 1. The substantive interest is the stability of alienation across time as represented by the path coefficient $\gamma_{3}$. Notice that, in Figure $1, e_{3}$ and $e_{5}$ are correlated and so are $e_{4}$ and $e_{6}$, which are justified by the nature of longitudinal data. However, correlated errors are not encouraged in general (see MacCallum, Roznowski, \& Necowitz, 1992). It is interesting to know the effect of excluding the error covariances on $\gamma_{3}$. Of course, one may fit the models with and without the correlated errors to Wheaton et al.'s (1977) data and compare the parameter estimates. Due to sampling error however, the change in parameter estimates may not give us a clear picture of the population value. Also, comparing the estimates does not facilitate a good understanding of the interrelation of all the paths in Figure 1. The technique of analyzing the path will allow us to predict the direction of parameter change before actually running the model. It also allows us to thoroughly understand the interrelation among all the model parameters. We return to this example after presenting the technique of analyzing the path.

Although there exist many methods for parameter estimation (Yuan \& Bentler, 2007b), the normal distribution-based maximum likelihood (ML) is the default 


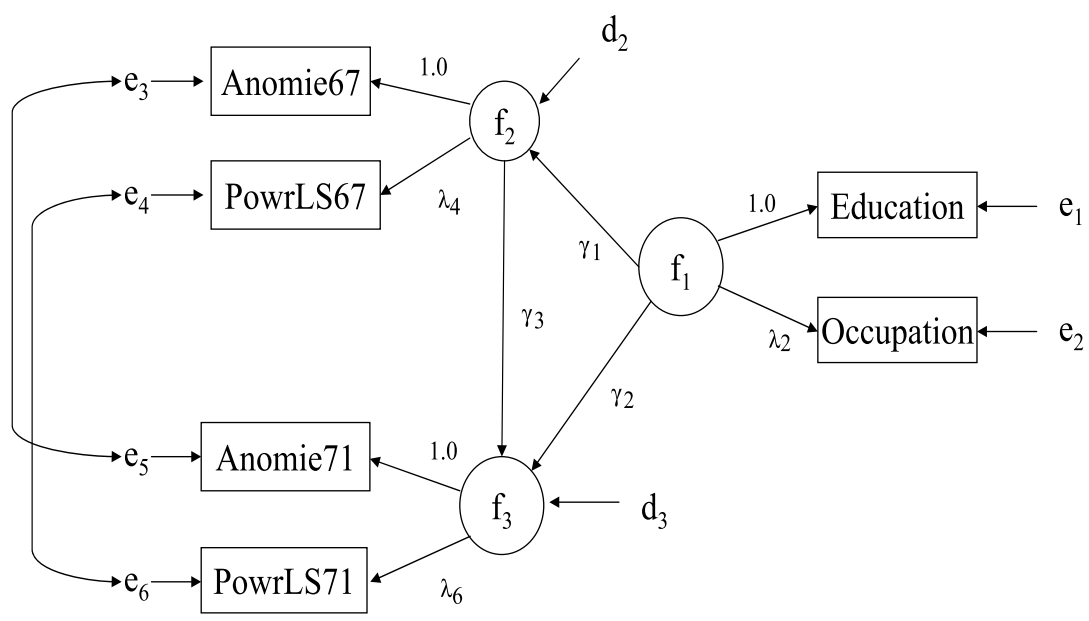

FIGURE 1 The path diagram for modeling the stability of alienation.

procedure in essentially all SEM programs (Amos, EQS, LISREL, Mplus, Mx, SAS Calis, sem) and also the most commonly used procedure in practice. We mainly consider biases in the ML estimates. Throughout the text we denote $\mathbf{S}=\left(s_{i j}\right)$ as the unbiased sample counterparts of $\boldsymbol{\Sigma}$ and $\mathbf{M}=\left(m_{i j}\right)$ as the modelimplied covariance matrix. For covariances involving latent variables, we use subscripts to distinguish them from the covariances between manifest variables as in $m_{x_{1} f_{1}}$ and $\sigma_{e_{1} e_{2}}$. We use $\lambda, \phi, \psi, \gamma$, and $\varphi$ for the parameter of factor loadings, factor variances and covariances, error variances and covariances, path coefficients between latent variables, and the variances of disturbances, respectively. Their estimates will be $\hat{\lambda}, \hat{\phi}, \hat{\psi}, \hat{\gamma}$, and $\hat{\varphi}$. The population value of the parameter $\boldsymbol{\theta}$ corresponding to model $\mathbf{M}(\boldsymbol{\theta})$ will be denoted as $\boldsymbol{\theta}_{*}$ in general and as $\boldsymbol{\theta}_{0}$ when $\boldsymbol{\Sigma}=\mathbf{M}\left(\boldsymbol{\theta}_{0}\right)$. For example, $\lambda_{10}$ is the value for factor loading $\lambda_{1}$ in a correctly specified model and $\lambda_{1 *}$ is the value of $\lambda_{1}$ in a misspecified model. When the model is misspecified, $\hat{\boldsymbol{\theta}}$ will approach $\boldsymbol{\theta}_{*}$ as the sample size $N$ increases. For a parameter $\theta$, its estimate $\hat{\theta}$ will be different from $\theta_{0}$ in general, the systematic difference or bias is $\theta_{*}-\theta_{0}$, and the difference due to sampling errors or a finite sample size is $\hat{\theta}-\theta_{*}$. Because $\hat{\theta}$ always contains sampling error, even for a correctly specified model, we discuss the change in $\theta_{*}$ when a model is misspecified. We always compare $\theta_{*}$ to $\theta_{0}$. A greater $\theta_{*}$ means $\theta_{*}-\theta_{0}>0$ and a smaller one means $\theta_{*}-\theta_{0}<0$. Thus, a greater $\theta_{*}$ implies $\hat{\theta}$ contains a positive bias, whereas a smaller $\theta_{*}$ implies $\hat{\theta}$ contains a negative bias. The biases in $\hat{m}=m(\hat{\boldsymbol{\theta}})$ will be $m_{*}-\sigma$; for example, $m_{12 *}-\sigma_{12}$ is the bias in $\hat{m}_{12}$. 
An intact parameter, variance, or covariance means the value of the parameter, variance, or covariance remains the same when the model changes.

Our aim is to illustrate the technique of analyzing the path using simple confirmatory factor models and SEM models so that readers will master the technique and effectively apply it in model diagnosis. The next section studies the effect of model misspecification on parameter biases in two confirmatory factor models where analyzing the path is straightforward. After that, we study the same effect in two structural equation models where the model may involve direct and indirect effects. Then, the analysis becomes relatively more complicated and we also use basic covariance algebra to facilitate the analysis. Finally, we apply the developed technique to study the change in $\gamma_{3}$ in Figure 1 and two other models based on real data that have been previously reported in the literature. Because the commonly used path diagrams and models being estimated correspond to unstandardized parameters, we mainly study the biases in unstandardized parameter estimates. Biases in standardized coefficients as well as conditions that may interfere with the results predicted by analyzing the path are discussed in the concluding section. The analysis is closely related to path diagrams, but only a limited number of them are presented to save space. Readers may draw additional path diagrams, using dashed one-way or two-way arrows to represent omitted paths, which will facilitate the understanding of the analysis.

\section{CONFIRMATORY FACTOR MODELS}

We consider a one-factor model and a three-factor model. The same analysis can be applied to other factor models. We only explicitly discuss cases when the excluded path bears a positive loading. With a negative loading, parameters will change in the opposite direction of those corresponding to a positive loading, as implied by Equation 1.

\section{A One-Factor Model}

In a one-factor model, excluded paths can only occur between errors. ${ }^{3}$ Suppose two errors are correlated and the model is correctly specified when the error covariance is explicitly modeled. Due to the ideal for unidimensional measurements (see Anderson \& Gerbing, 1988), the model is set without any correlated errors. We study the effect of such a misspecification by first considering the

\footnotetext{
${ }^{3}$ Paths between indicators and errors may also exist (see Bentler, 1995, p. 103). These are seldom employed in practice and we do not consider them here.
} 
case where the factor variance is set to 1 (i.e., $\phi_{11}=m_{f_{1} f_{1}}=1$ ), and then considering setting a path loading to 1 (i.e., $\lambda_{1}=1$ ). Both specifications are commonly used for model identification.

In Figure 2, the variance of the factor is set equal to $1\left(\phi_{11}=1\right)$ and the errors $e_{1}$ and $e_{2}$ are positively correlated in the population $\left(\sigma_{e_{1} e_{2}}>0\right)$; however the path $e_{1} \leftrightarrow e_{2}$ is excluded. The model needs to explain the extra association between $x_{1}$ and $x_{2}$. The only path for such an association is $x_{1} \leftarrow f_{1} \rightarrow x_{2}$ with the model-implied covariance given by $m_{12}=\lambda_{1} \lambda_{2}$. Thus, $\lambda_{1 *} \lambda_{2 *}$ has to be greater than the population counterpart $\lambda_{10} \lambda_{20}$ corresponding to the correctly specified model. Due to the parallel positions of $\lambda_{1}$ and $\lambda_{2}$ in Figure 2, we must have $\lambda_{1 *}>\lambda_{10}$ and $\lambda_{2 *}>\lambda_{20}$. In the estimation of the factor model represented by Figure 2, the distance between all the pairs of $(s, m)$ is minimized simultaneously. In estimating $\lambda_{1}$ and $\lambda_{2}$, the system of equations also estimates $\lambda_{3}, \lambda_{4}$, and $\psi_{11}$ to $\psi_{44}$. With the greater $\lambda_{1 *}$ and $\lambda_{2 *}$, it is necessary to have $\lambda_{3 *}<\lambda_{30}$ to explain $\sigma_{13}$ and $\sigma_{23}$. Similarly, $\lambda_{4 *}<\lambda_{40}$ needs to hold to explain $\sigma_{14}$ and $\sigma_{24}$. Ideally, it needs $\lambda_{3 *} \lambda_{4 *}=\lambda_{30} \lambda_{40}$ to fully explain $\sigma_{34}$, which is unlikely due to the misspecification. Because the unique variances in Figure 2 are free parameters, they can take any values to explain the variances $\sigma_{i i}$, $i=1$ to 4 . Because $\lambda_{1 *}>\lambda_{10}, \lambda_{2 *}>\lambda_{20}, \lambda_{3 *}<\lambda_{30}$, and $\lambda_{4 *}<\lambda_{40}$, we have $\psi_{11 *}<\psi_{110}, \psi_{22 *}<\psi_{220}, \psi_{33 *}>\psi_{330}$ and $\psi_{44 *}>\psi_{440}$. Actually, in the ML procedure, $\lambda_{i *}$ and $\psi_{i i *}$ satisfy $\lambda_{i *}^{2}+\psi_{i i *}=\sigma_{i i}$. A greater $\lambda_{i *}$ implies

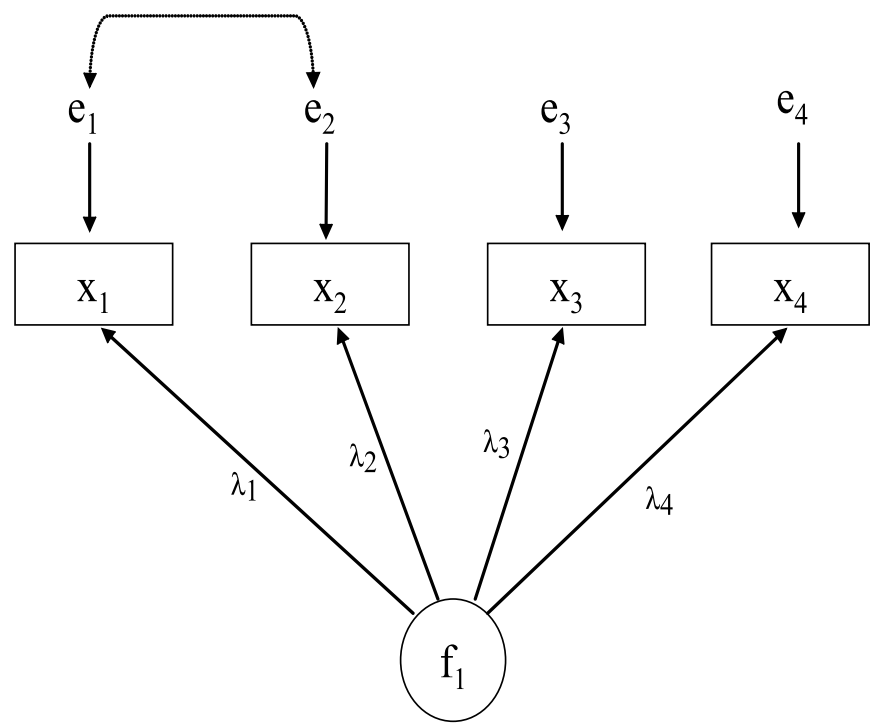

FIGURE 2 The path diagram for a one-factor model with $\phi_{11}=1$. 
a smaller $\psi_{i i *}$ and vice versa. ${ }^{4}$ The results of the analysis for Figure 2 are summarized by

\begin{tabular}{cccccccc}
\hline$\lambda_{1 *}$ & $\lambda_{2 *}$ & $\lambda_{3 *}$ & $\lambda_{4 *}$ & $\psi_{11 *}$ & $\psi_{22 *}$ & $\psi_{33 *}$ & $\psi_{44 *}$ \\
+ & + & - & - & - & - & + & + \\
\hline
\end{tabular}

where + means greater and - means smaller. In summary, when two errors in Figure 2 are positively correlated in the population, the factor loadings corresponding to the variables will be positively biased, and the error variances will be negatively biased. The other factor loadings will be negatively biased and error variances will be positively biased.

When $\lambda_{1}=1$ is used for model identification as in Figure 3 , the $\lambda_{i 0}$ or $\lambda_{i *}$ will be generally different from those in Figure 2 for the given $\sigma$ 's, but they are still uniquely defined (see Steiger, 2002). When $\sigma_{e_{1} e_{2}}>0$ and $e_{1} \leftrightarrow e_{2}$ is not included, the path $x_{1} \leftarrow f_{1} \rightarrow x_{2}$ has to explain the extra association using $m_{12}=\lambda_{2} \phi_{11}$. Thus, $\lambda_{2 *} \phi_{11 *}>\lambda_{20} \phi_{110}$. Notice that $m_{23}=\lambda_{2} \lambda_{3} \phi_{11}$ and $m_{24}=\lambda_{2} \lambda_{4} \phi_{11}$. The greater $\lambda_{2 *} \phi_{11 *}$ leads to $\lambda_{3 *}<\lambda_{30}$ and $\lambda_{4 *}<\lambda_{40}$. Due to $m_{13}=\lambda_{3} \phi_{11}, m_{14}=\lambda_{4} \phi_{11}$ and smaller $\lambda_{3 *}$ and $\lambda_{4 *}, \phi_{11 *}$ and $\phi_{110}$ satisfy $\phi_{11 *}>\phi_{110}$. It might seem that $\lambda_{2 *}$ can be greater or smaller. Comparing $m_{34}=\lambda_{3} \lambda_{4} \phi_{11}$ with $m_{23}=\lambda_{2} \lambda_{3} \phi_{11}$ and $m_{24}=\lambda_{2} \lambda_{4} \phi_{11}$, where the positions of $\lambda_{2}, \lambda_{3}$, and $\lambda_{4}$ are parallel in modeling the intact $\sigma_{34}, \sigma_{23}$, and $\sigma_{24}, \lambda_{2 *}$ will most likely be smaller. Because $m_{12 *}$ will increase more than $m_{13 *}$ and $m_{14 *}$, $\lambda_{2 *}-\lambda_{20}$ may not be as small as $\lambda_{3 *}-\lambda_{30}$ or $\lambda_{4 *}-\lambda_{40}$. It is mainly $\phi_{11 *}$ that will take the greater association between $x_{1}$ and $x_{2}$. Because $\phi_{11 *}>\phi_{110}, \psi_{11 *}$ will be smaller. Our analysis cannot predict the directions of change in $\psi_{22 *}$, $\psi_{33 *}$, and $\psi_{44 *}$.

When $\sigma_{e_{2} e_{3}}>0$ and $e_{2} \leftrightarrow e_{3}$ is excluded in Figure 3, the path $x_{2} \leftarrow f_{1} \rightarrow x_{3}$ has to explain the extra association using $m_{23}=\lambda_{2} \lambda_{3} \phi_{11}$, at least one of $\lambda_{2 *}$, $\lambda_{3 *}$, and $\phi_{11 *}$ should be greater. Notice that $\phi_{11}$ is involved in every modelimplied covariance, hence a greater $\phi_{11}^{*}$ will not solve the problem. Due to their parallel position, $\lambda_{2 *}$ and $\lambda_{3 *}$ will both be greater. Greater $\lambda_{2 *}$ and $\lambda_{3 *}$ need a smaller $\phi_{11 *}$ to explain $\sigma_{12}$ by $m_{12 *}=\lambda_{2 *} \phi_{11 *}$ and $\sigma_{13}$ by $m_{13 *}=\lambda_{3 *} \phi_{11 *}$. The parameter $\lambda_{4}$ is involved in the model-implied covariances $m_{14}, m_{24}$, and $m_{34}$, which require $\lambda_{4 *}$ be greater, smaller, and smaller, respectively. So $\lambda_{4 *}$ will most likely become smaller. Smaller $\phi_{11 *}$ and $\lambda_{4 *}$ imply greater $\psi_{11 *}$ and $\psi_{44 *}$. Notice that $\lambda_{2 *} \lambda_{3 *} \phi_{11 *}$ is greater and the positions of $\lambda_{2}$ and $\lambda_{3}$ are parallel in reacting to the misspecification, hence $\lambda_{2 *}^{2} \phi_{11 *}$ and $\lambda_{3 *}^{2} \phi_{11 *}$ should both be

\footnotetext{
${ }^{4}$ For commonly used models, the diagonal elements of the residual covariance matrix are all zero (Shapiro \& Browne, 1990). When the diagonal elements of the residual covariance matrix are not zero, a greater factor loading may not imply a smaller error variance.
} 


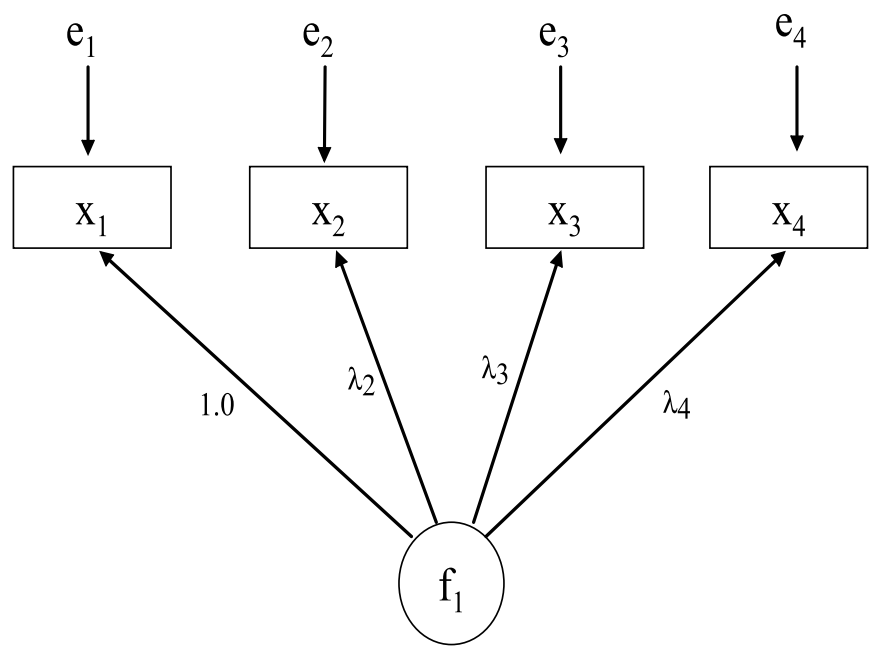

FIGURE 3 The path diagram for a one-factor model with $\phi_{11}$ being a free parameter.

greater and $\psi_{22 *}$ and $\psi_{33 *}$ should become smaller. The analysis for Figure 3 is summarized as

\begin{tabular}{c|cccccccc}
\hline$\theta_{*}$ & $\phi_{11 *}$ & $\lambda_{2 *}$ & $\lambda_{3 *}$ & $\lambda_{4 *}$ & $\psi_{11 *}$ & $\psi_{22 *}$ & $\psi_{33 *}$ & $\psi_{44 *}$ \\
$\sigma_{e_{1} e_{2}}>0$ & + & - & - & - & - & $?$ & $?$ & $?$ \\
$\sigma_{e_{2} e_{3}}>0$ & - & + & + & - & + & - & - & + \\
\hline
\end{tabular}

where? implies our analysis cannot determine the direction of the change.

For the simple case of a one-factor model, analyzing the path clearly shows the effect of excluding error covariances on other parameters in the model. The parameter estimates corresponding to + will have positive biases and those corresponding to - will have negative biases.

\section{A Confirmatory Three-Factor Model}

There are two kinds of misspecifications for a three-factor model. One is when error covariances are excluded, and the other is when factor loadings are excluded. We analyze the effect of excluding correlated errors first and then the effect of excluding factor loadings. We only consider the case when all the factor variances are set at $1.0\left(\phi_{j j}=1\right)$ in analyzing the model. Cases when one, two, or three $\lambda_{i} \mathrm{~s}$ are fixed for identification can be analyzed similarly. The indicators for a factor are called a cluster. 
Figure 4 is a path diagram for a confirmatory factor model with three factors and nine indicators. Yuan et al. (2003) analyzed the effect of $\sigma_{e_{1} e_{2}}>0$ on parameter estimates in this model. The analysis is similar to the one-factor model in Figure 2. We summarize their results without repeating the analysis. When $\sigma_{e_{1} e_{2}}>0$ in Figure $4, \lambda_{1 *}$ and $\lambda_{2 *}$ will be greater; $\lambda_{3 *}$ will be smaller; $\psi_{11 *}$ and $\psi_{22 *}$ will be smaller; $\psi_{33 *}$ will be greater; and $\phi_{12 *}$ and $\phi_{13 *}$ tend to be smaller. The parameters $\lambda_{4 *}$ to $\lambda_{9 *}, \phi_{23 *}$, and $\psi_{44 *}$ to $\psi_{99 *}$ will equal their counterparts in a correctly specified model. Due to the parallel positions of the variables, the effect of any $\sigma_{e_{i} e_{j}}>0$ within a cluster in Figure 4 can be obtained similarly (see Yuan et al., 2003, pp. 247-248).

For correlated errors corresponding to indicators for different factors, we only consider $\sigma_{e_{1} e_{4}}>0$ in Figure 4 due to the parallel positions of the variables. The only path that can explain the greater $\sigma_{14}$ in Figure 4 is $x_{1} \leftarrow f_{1} \leftrightarrow f_{2} \rightarrow x_{4}$. The $\lambda_{i}$ s need to account for the within-cluster associations, so $\phi_{12 *}$ has to be greater. Because $m_{14 *}=\lambda_{1 *} \lambda_{4 *} \phi_{12 *}$ needs to be greater, $m_{25 *}, m_{26 *}, m_{35 *}$, and $m_{36 *}$ do not need to be; $\lambda_{1 *}$ and $\lambda_{4 *}$ will be greater. Because the pairwise

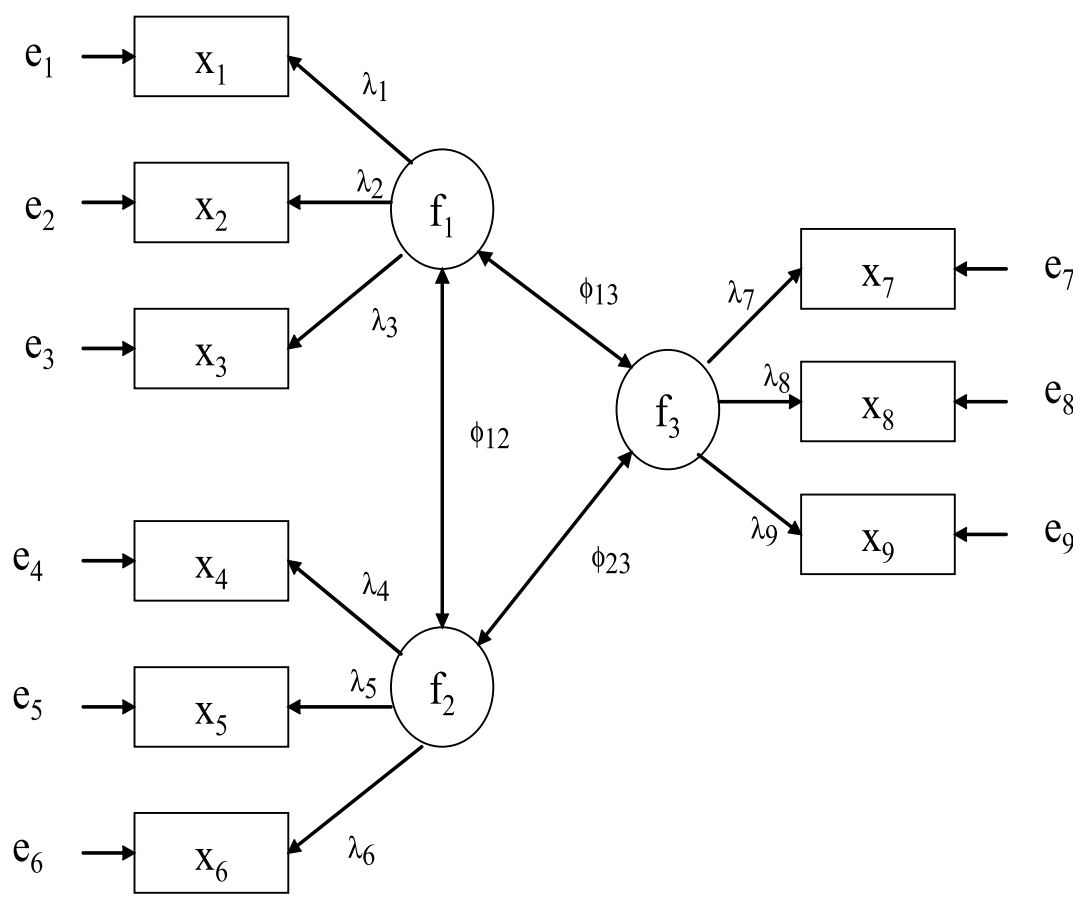

FIGURE 4 The path diagram for a three-factor model with $\phi_{j j}=1$. 
population covariances within clusters $\left(x_{1}, x_{2}, x_{3}\right)$ and $\left(x_{4}, x_{5}, x_{6}\right)$ remain the same, factor loadings $\lambda_{2 *}, \lambda_{3 *}, \lambda_{5 *}$, and $\lambda_{6 *}$ have to be smaller to adjust for the greater $\lambda_{1 *}$ and $\lambda_{4 *}$. The parameter $\psi_{i i}$ needs to adjust for the difference between the model-implied variance; $\sigma_{i i}, \psi_{11 *}$, and $\psi_{44 *}$ have to be smaller; and $\psi_{22 *}, \psi_{33 *}, \psi_{55 *}$, and $\psi_{66 *}$ have to be greater. Being responsible for the between-cluster covariances of $\left(x_{1}, x_{2}, x_{3}\right)$ and $\left(x_{4}, x_{5}, x_{6}\right)$ with $\left(x_{7}, x_{8}, x_{9}\right)$, $\phi_{13 *}$ and $\phi_{23} *$ will be affected due to the changes of factor loadings within $\left(x_{1}, x_{2}, x_{3}\right)$ and $\left(x_{4}, x_{5}, x_{6}\right)$, but we are unable to determine the direction of their changes. The parameters $\lambda_{7}$ to $\lambda_{9}$ explain the within-cluster associations among $x_{7}$ to $x_{9}$, and their values will not affect the between-cluster covariances of $\left(x_{1}, x_{2}, x_{3}\right)$ and $\left(x_{4}, x_{5}, x_{6}\right)$. The effect of the misspecification is accounted for by $\phi_{13 *}$ and $\phi_{23 *}$ before reaching $\left(x_{7}, x_{8}, x_{9}\right)$, thus, $\lambda_{7 *}$ to $\lambda_{9 *}$ and $\psi_{77 *}$ to $\psi_{99 *}$ will equal their population values corresponding to the correctly specified model.

TABLE 1

The Directions of Change in Parameters When a Positive Error Covariance or Extra Factor Loading

Exists in the Population and the Model is

Represented by Figure 4

\begin{tabular}{cccc}
\hline$\theta_{*}$ & $\sigma_{e_{1} e_{2}}$ & $\sigma_{e_{\text {e }} 4}$ & $\lambda_{320}$ \\
\hline$\lambda_{1 *}$ & + & + & - \\
$\lambda_{2 *}$ & + & - & - \\
$\lambda_{3 *}$ & - & - & + \\
$\lambda_{4 *}$ & 0 & + & 0 \\
$\lambda_{5 *}$ & 0 & - & 0 \\
$\lambda_{6 *}$ & 0 & - & 0 \\
$\lambda_{7 *}$ & 0 & 0 & 0 \\
$\lambda_{8 *}$ & 0 & 0 & 0 \\
$\lambda_{9 *}$ & 0 & 0 & 0 \\
$\phi_{12 *}$ & - & + & + \\
$\phi_{13 *}$ & - & $?$ & $?$ \\
$\phi_{23 *}$ & 0 & $?$ & 0 \\
$\psi_{11 *}$ & - & - & + \\
$\psi_{22 *}$ & - & + & + \\
$\psi_{33 *}$ & + & + & $?$ \\
$\psi_{44 *}$ & 0 & - & 0 \\
$\psi_{55 *}$ & 0 & + & 0 \\
$\psi_{66 *}$ & 0 & + & 0 \\
$\psi_{77 *}$ & 0 & 0 & 0 \\
$\psi_{88 *}$ & 0 & 0 & 0 \\
$\psi_{99 *}$ & 0 & 0 & 0 \\
\hline
\end{tabular}


Notice that $x_{3}$ does not load on $f_{2}$ in Figure 4 . When the correct model needs $x_{3}$ to load on $f_{2}$ with a positive loading ${ }^{5} \lambda_{320}$, all the covariances between $x_{3}$ and the other variables $\left(\sigma_{3 i} \mathrm{~s}\right)$ are positively perturbed. The relation of $x_{3}$ with $\left(x_{4}, x_{5}, x_{6}\right)$ and $\left(x_{7}, x_{8}, x_{9}\right)$ needs to pass $f_{1} \leftrightarrow f_{2}$ and $f_{1} \leftrightarrow f_{3}$, which will be accounted for by $\phi_{12}$ and $\phi_{13}$, respectively. The between-cluster covariances of $\left(x_{4}, x_{5}, x_{6}\right)$ with $\left(x_{7}, x_{8}, x_{9}\right)$ are intact. To explain the intact covariances, we have $\lambda_{4 *}=\lambda_{40}, \ldots, \lambda_{9 *}=\lambda_{90}, \phi_{23 *}=\phi_{230}$, and $\psi_{44 *}=\psi_{440}, \ldots, \psi_{99 *}=\psi_{990}$. The model needs to mainly explain the perturbed $\sigma_{3 i}$ by $x_{3} \leftarrow f_{1} \leftrightarrow f_{2}$ with the model-implied covariance $m_{x_{3} f_{2}}=\lambda_{3} \phi_{12}$. So there must exist $\lambda_{3 *} \phi_{12 *}>$ $\lambda_{30} \phi_{120}$. Notice that it is $x_{3}$ that has a greater covariance with $f_{2}$, not $x_{1}$ or $x_{2}$, so $\lambda_{3 *}$ must be greater. When $\phi_{120}$ and $\phi_{130}$ are comparable, $\sigma_{x_{3} f_{2}}=\lambda_{3} \phi_{12}$ is positively perturbed more by the additional $\lambda_{320}>0$ than $\sigma_{x_{3} f_{3}}=\lambda_{3} \phi_{13}$, $\phi_{12 *}$ also needs to be greater to explain the greater perturbation. With a greater $\phi_{12 *}, \lambda_{1 *}$ and $\lambda_{2 *}$ need to be smaller to explain the intact covariances between $\left(x_{1}, x_{2}\right)$ and $\left(x_{4}, x_{5}, x_{6}\right)$; consequently, $\psi_{11 *}$ and $\psi_{22 *}$ will be greater. Because $\lambda_{320}>0$ also makes $\sigma_{33}$ greater, we cannot determine the direction of change in $\psi_{33 *}$. The parameter $\phi_{13}$ is responsible for the covariance between the clusters $\left(x_{1}, x_{2}, x_{3}\right)$ and $\left(x_{7}, x_{8}, x_{9}\right)$. Due to the change in $\lambda_{1 *}$ to $\lambda_{3 *}$, and the greater $\sigma_{3 i}, i=7$ to 9 , we cannot determine the direction of change in $\phi_{13 *}$. The results of the analysis for Figure 4 are summarized in Table 1.

\section{STRUCTURAL EQUATION MODELS}

We consider structural equation models with two and three factors. Similarities and differences between the two models allow us to understand the functions of similar parameters under different constraints. Compared to the previous section, the analysis for structural equation models is more complex. However, we employ the same technique as was used for the confirmatory factor models. That is, when excluding a path with a nonzero loading between two variables, the existing paths indirectly connecting these two variables in the model need to compensate. Loading estimates of the paths may have positive or negative biases. The technique of analyzing the path allows us to determine the directions of bias in many of these estimates.

\section{A Model With Two Factors}

Figure 5 is a path diagram of a simple structural equation model with six indicators and two factors, where $m_{f_{1} f_{1}}=\phi_{11}, m_{d_{2} d_{2}}=\varphi_{22}$, and $m_{e_{i} e_{i}}=\psi_{i i}$.

\footnotetext{
${ }^{5}$ When $\lambda$ has double nonzero subscripts, the first indicates the order of the variable and the second indicates the order of the factor.
} 


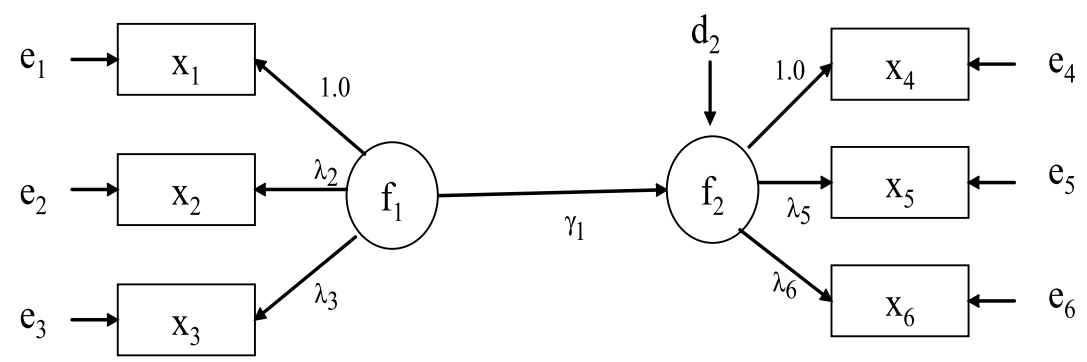

FIGURE 5 The path diagram for a structural equation model with two factors.

The model does not contain any error covariances and all the indicators are unidimensional. We consider the existence of positive covariances $\sigma_{e_{1} e_{2}}, \sigma_{e_{2} e_{3}}$, $\sigma_{e_{4} e_{5}}, \sigma_{e_{5} e_{6}}, \sigma_{e_{1} e_{4}}, \sigma_{e_{1} e_{5}}, \sigma_{e_{2} e_{4}}$, and $\sigma_{e_{2} e_{5}}$ and double loadings represented by $x_{1} \leftarrow f_{2}, x_{2} \leftarrow f_{2}, x_{4} \leftarrow f_{1}, x_{5} \leftarrow f_{1}$ in the population. The loading parameters will be denoted by $\lambda_{12}, \lambda_{22}, \lambda_{41}$, and $\lambda_{51}$, respectively. For the same reason as with the three-factor model in the previous section, when a pair of errors within a cluster are correlated and the correlation is excluded from the model, the relation within the other cluster is still correctly evaluated. Similarly, when $\lambda_{i 20}>0$, $i=1,2,3$, the relation within the cluster $\left(x_{4}, x_{5}, x_{6}\right)$ is not affected; when $\lambda_{i 10}>0, i=4,5,6$, the relation within the cluster $\left(x_{1}, x_{2}, x_{3}\right)$ is not affected either. The results are summarized in Table 2 . Now readers may analyze Figure 5 and compare their results with those in Table 2. To avoid repetition, we only

TABLE 2

The Directions of Change in Parameters When a Positive Error Covariance or Extra Factor Loading Exists in the Population and the Model is Represented by Figure 5

\begin{tabular}{ccccccccccccc}
\hline$\theta_{*}$ & $\sigma_{e_{1} e_{2}}$ & $\sigma_{e_{2} e_{3}}$ & $\sigma_{e_{4} e_{5}}$ & $\sigma_{e_{5} e_{6}}$ & $\sigma_{e_{1} e_{4}}$ & $\sigma_{e_{1} e_{5}}$ & $\sigma_{e_{2} e_{4}}$ & $\sigma_{e_{2} e_{5}}$ & $\lambda_{120}$ & $\lambda_{220}$ & $\lambda_{410}$ & $\lambda_{510}$ \\
\hline$\lambda_{2 *}$ & - & + & 0 & 0 & - & - & + & + & - & + & 0 & 0 \\
$\lambda_{3 *}$ & - & + & 0 & 0 & - & - & - & - & - & $?$ & 0 & 0 \\
$\lambda_{5 *}$ & 0 & 0 & - & + & - & + & - & + & 0 & 0 & - & + \\
$\lambda_{6 *}$ & 0 & 0 & - & + & - & - & - & - & 0 & 0 & - & - \\
$\phi_{11 *}$ & + & - & 0 & 0 & + & + & - & - & + & - & 0 & 0 \\
$\gamma_{1 *}$ & - & - & + & - & $?$ & $?$ & + & + & - & + & + & + \\
$\varphi_{22 *}$ & $?$ & + & + & $?$ & - & - & - & - & - & - & $?$ & $?$ \\
$\psi_{11 *}$ & - & + & 0 & 0 & - & - & + & + & $?$ & + & 0 & 0 \\
$\psi_{22 *}$ & $?$ & $?$ & 0 & 0 & $?$ & $?$ & - & - & $?$ & $?$ & 0 & 0 \\
$\psi_{33 *}$ & $?$ & $?$ & 0 & 0 & $?$ & $?$ & $?$ & $?$ & $?$ & $?$ & 0 & 0 \\
$\psi_{44 *}$ & 0 & 0 & - & + & - & $?$ & $?$ & $?$ & 0 & 0 & $?$ & $?$ \\
$\psi_{55 *}$ & 0 & 0 & $?$ & $?$ & $?$ & - & $?$ & - & 0 & 0 & $?$ & $?$ \\
$\psi_{66 *}$ & 0 & 0 & $?$ & $?$ & $?$ & $?$ & $?$ & $?$ & 0 & 0 & $?$ & $?$ \\
\hline
\end{tabular}


discuss parameters that are affected and provide the outline for the analyses that lead to the results.

When $\sigma_{e_{1} e_{2}}>0$ in Figure 5, $m_{12 *}=\lambda_{2 *} \phi_{11 *}$ has to be greater to account for the extra association between $x_{1}$ and $x_{2}$. Because $m_{23}=\lambda_{3} m_{12}, \lambda_{3 *}$ has to be smaller to account for the greater $m_{12 *}$. A smaller $\lambda_{3 *}$ needs a greater $\phi_{11 *}$ to explain the intact $\sigma_{31}$. Greater $\phi_{11 *}$ and $\left(m_{x_{1} f_{1} *}+m_{x_{2} f_{1} *}+m_{x_{3} f_{1} *}\right) / 3=(1+$ $\left.\lambda_{2 *}+\lambda_{3 *}\right) \phi_{11 *} / 3$ need a smaller $\gamma_{1 *}$ for the intact between-cluster covariances. It might seem that $\lambda_{2 *}$ can be smaller or greater. However, due to a greater $\phi_{11 *}$, similar to $\lambda_{3 *}, \lambda_{2 *}$ should be smaller to explain the intact between-cluster covariances. A greater $\phi_{11 *}$ leads to a smaller $\psi_{11 *}$. We are unable to predict the directions of change in $\varphi_{22 *}, \psi_{22 *}$, and $\psi_{33 *}$. When a parameter $\theta_{1}$ is closely related to two other parameters $\theta_{2}$ and $\theta_{3}$, if $\theta_{2 *}$ needs a smaller $\theta_{1 *}$ and $\theta_{3 *}$ needs a greater $\theta_{1 *}$, we will not be able to predict the direction of change in $\theta_{1 *}$ in general.

When $\sigma_{e_{2} e_{3}}>0$, the path $x_{2} \leftarrow f_{1} \rightarrow x_{3}$ is responsible for explaining the greater $\sigma_{23}$ with $m_{23}=\lambda_{2} \lambda_{3} \phi_{11}$. Due to the parallel positions of $\lambda_{2}$ and $\lambda_{3}$ in the model, both of them have to be either greater or smaller. Notice that $\lambda_{2}=m_{23} / m_{13}, \lambda_{3}=m_{23} / m_{12}$, and $\sigma_{13}$ and $\sigma_{12}$ are intact, $\lambda_{2 *}$ and $\lambda_{3 *}$ have to be greater. Due to greater $\lambda_{2 *}$ and $\lambda_{3 *}, \phi_{11 *}$ has to be smaller, which leads to a greater $\psi_{11 *}$. Similarly, the $\left(m_{x_{1} f_{1} *}+m_{x_{2} f_{1} *}+m_{x_{3} f_{1} *}\right) / 3$ is greater due to the stronger within-cluster associations, which leads to a smaller $\gamma_{1 *}$ for the intact between-cluster associations. A smaller $\gamma_{1 *}$ together with a smaller $\phi_{11 *}$ needs a greater $\varphi_{22 *}$ for the intact $m_{f_{2} f_{2}}$. We are unable to predict the directions of change in $\psi_{22 *}$ and $\psi_{33 *}$.

When $\sigma_{e_{4} e_{5}}>0, m_{45 *}=\lambda_{5 *} m_{f_{2} f_{2} *}$ has to be greater. Because $m_{56}=\lambda_{6} m_{45}$, $\lambda_{6 *}$ has to be smaller. Parallel to $\lambda_{6 *}, \lambda_{5 *}$ needs to be smaller for the intact between-cluster covariances. Smaller $\lambda_{5 *}$ and $\lambda_{6 *}$ need a greater $m_{f_{2} f_{2} *}$. The only function of $\varphi_{22 *}$ is to adjust for $m_{f_{2} f_{2} *}$; a greater $m_{f_{2} f_{2} *}$ needs a greater $\varphi_{22 *}$. Notice that only $\phi_{11}$ and $\gamma_{1}$, not $\varphi_{22 *}$, contribute to the between-cluster covariances, hence smaller $\lambda_{5 *}$ and $\lambda_{6 *}$ need a greater $\gamma_{1 *}$. A greater $m_{f_{2} f_{2} *}$ leads to a smaller $\psi_{44 *}$. We are unable to predict the directions of change in $\psi_{55 *}$ and $\psi_{66 *}$.

When $\sigma_{e_{5} e_{6}}>0, m_{56 *}=\lambda_{5 *} \lambda_{6 *} m_{f_{2} f_{2} *}$ has to be greater. Due to their parallel positions, both $\lambda_{5 *}$ and $\lambda_{6 *}$ have to be greater. Greater $\lambda_{5 *}$ and $\lambda_{6 *}$ need a smaller $\gamma_{1 *}$ to explain the intact between-cluster covariances and a smaller $m_{f_{2} f_{2} *}$ to explain $\sigma_{45}$ and $\sigma_{46}$. A smaller $m_{f_{2} f_{2} *}$ leads to a greater $\psi_{44 *}$. We are unable to predict the directions of change in $\varphi_{22 *}, \psi_{55 *}$, and $\psi_{66 *}$.

When $\sigma_{e_{1} e_{4}}>0, m_{14 *}=m_{f_{1} f_{2} *}=\phi_{11 *} \gamma_{1 *}$ has to be greater. There are three possibilities: (a) $\gamma_{1 *}$ is much greater and $\phi_{11 *}$ is slightly smaller or greater; (b) $\phi_{11 *}$ is much greater and $\gamma_{1 *}$ is slightly smaller or greater; and (c) both $\phi_{11 *}$ and $\gamma_{1 *}$ are greater. Because the only function of $\varphi_{22 *}$ is to adjust for $m_{f_{2} f_{2} *}$, the values of $\phi_{11 *}$ and $\gamma_{1 *}$ will not affect the associations within the cluster 
$\left(x_{4}, x_{5}, x_{6}\right)$. Under (a), $\lambda_{2 *}$ and $\lambda_{3 *}$ need to be much smaller to explain the between-cluster covariances and they need to remain approximately the same to explain the covariances within $\left(x_{1}, x_{2}, x_{3}\right)$, so (a) is unlikely to happen. Under (c), $\lambda_{2 *}$ and $\lambda_{3 *}$ only need to adjust for the effect of a greater $\phi_{11 *}$ for the intact covariances within $\left(x_{1}, x_{2}, x_{3}\right)$ and they need to adjust for both the effect of a greater $\phi_{11 *}$ and a greater $\gamma_{1 *}$ in modeling the intact $\sigma_{24}, \sigma_{25}, \sigma_{26}$, and $\sigma_{34}$, $\sigma_{35}, \sigma_{36}$, so (c) is unlikely to happen either. Notice that all the model-implied covariances contain $\phi_{11}$. Except for $m_{14}$, all also contain at least a $\lambda_{i}$. The most likely scenario is therefore (b). Thus, $\phi_{11 *}$ should be greater, $\lambda_{2 *}, \lambda_{3 *}, \lambda_{5 *}$ and $\lambda_{6 *}$ will be smaller; $\psi_{11 *}$ will be smaller. Smaller $\lambda_{5 *}$ and $\lambda_{6 *}$ lead to a greater $m_{f_{2} f_{2} *}$ and thus, a smaller $\psi_{44 *}$. A greater $\phi_{11 *} \gamma_{1 *}$ also leads to a smaller $\varphi_{22 *}$. We are unable to determine the directions of change in $\gamma_{1 *}, \psi_{22 *}, \psi_{33 *}, \psi_{55 *}$, and $\psi_{66 *}$.

When $\sigma_{e_{1} e_{5}}>0, m_{15 *}=\lambda_{5 *} \phi_{11 *} \gamma_{1 *}$ has to be greater. Because $\phi_{11 *} \gamma_{1 *}$ is shared by all the between-cluster covariances, $\lambda_{5 *}$ should be much greater. A smaller $\phi_{11 *} \gamma_{1 *}$ is not supported by any of the between-cluster covariances either. A greater $m_{15 *}$ needs smaller $\lambda_{2 *}$ and $\lambda_{3 *}$ for the intact between-cluster covariances of $\left(x_{2}, x_{3}\right)$ with $\left(x_{4}, x_{5}, x_{6}\right)$. Smaller $\lambda_{2 *}$ and $\lambda_{3 *}$ lead to a greater $\phi_{11 *}$, which further leads to a smaller $\psi_{11 *}$. Smaller $\lambda_{2 *}$ and $\lambda_{3 *}$ also support a greater $\phi_{11 *} \gamma_{1 *}$. A smaller $\lambda_{6 *}$ is necessary to explain the intact $\sigma_{16}$ and $\sigma_{56}$. A greater $\lambda_{5 *}$ most likely leads to a smaller $\psi_{55 *}$. A greater $\phi_{11 *} \gamma_{1 *}$ also needs a smaller $\varphi_{22 *}$ for the intact covariances within $\left(x_{4}, x_{5}, x_{6}\right)$. We are unable to determine the directions of change in $\gamma_{1 *}, \psi_{22 *}, \psi_{33 *}, \psi_{44 *}$, and $\psi_{66 *}$.

When $\sigma_{e_{2} e_{4}}>0$, parallel to $\sigma_{e_{1} e_{5}}>0, \lambda_{2 *}$ is much greater and $\phi_{11 *} \gamma_{1 *}$ is also greater; $\lambda_{3 *}, \lambda_{5 *}$, and $\lambda_{6 *}$ are smaller. A much greater $\lambda_{2 *}$ needs a smaller $\phi_{11 *}$ for the intact covariances within $\left(x_{1}, x_{2}, x_{3}\right)$. A smaller $\phi_{11 *}$ needs a greater $\gamma_{1 *}$ and a greater $\psi_{11 *}$. A much greater $\lambda_{2 *}$ also most likely leads to a smaller $\psi_{22 *}$. A greater $\phi_{11 *} \gamma_{1 *}$ needs a smaller $\varphi_{22 *}$ for the intact covariances within $\left(x_{4}, x_{5}, x_{6}\right)$. We are unable to determine the directions of change in $\psi_{33 *}, \psi_{44 *}$, to $\psi_{66 *}$.

When $\sigma_{e_{2} e_{5}}>0, m_{25 *}=\lambda_{2 *} \lambda_{5 *} \phi_{11 *} \gamma_{1 *}$ has to be greater. Because $\phi_{11 *} \gamma_{1 *}$ is responsible for all the between-cluster covariances, $\lambda_{2 *}$ and $\lambda_{5 *}$ have to be greater. There is no reason for $\phi_{11 *} \gamma_{1 *}$ to be smaller either. A greater $\lambda_{2 *}$ needs a smaller $\phi_{11 *}$ for the intact covariances within $\left(x_{1}, x_{2}, x_{3}\right)$. Smaller $\lambda_{3 *}$ and $\lambda_{6 *}$ are also necessary for explaining the between-cluster associations. Greater $\lambda_{2 *}$ and $\lambda_{5 *}$ also most likely lead to smaller $\psi_{22 *}$ and $\psi_{55 *}$. A smaller $\phi_{11 *}$ needs a greater $\gamma_{1 *}$ and a greater $\psi_{11 *}$. We are unable to determine the directions of change in $\psi_{33 *}, \psi_{44 *}$, and $\psi_{66 *}$.

In Figure 5, $x_{1}$ does not load on $f_{2}$. If the correct model needs an extra path $x_{1} \leftarrow f_{2}$ with loading $\lambda_{120}>0$, the stronger association between $x_{1}$ and $f_{2}$ has to be explained by $m_{x_{1} f_{2}}=\gamma_{1} \phi_{11}$, thus $\gamma_{1 *} \phi_{11 *}$ will be greater. Notice that $m_{x_{2} f_{2}}=\lambda_{2} m_{x_{1} f_{2}}$ and $m_{x_{3} f_{2}}=\lambda_{3} m_{x_{1} f_{2}}$, hence $\lambda_{2 *}$ and $\lambda_{3 *}$ have to be smaller to explain the associations of $x_{2}$ and $x_{3}$ with the cluster $\left(x_{4}, x_{5}, x_{6}\right)$. Due to 
smaller $\lambda_{2 *}$ and $\lambda_{3 *}, \phi_{11 *}$ has to be much greater to explain the intact $\sigma_{23}$ and the extra associations of $x_{1}$ with $x_{2}$ and $x_{3}$. Notice that $m_{f_{2} f_{2} *}$ is not affected by the misspecification, and $\gamma_{1}$ is the path coefficient of $f_{1} \rightarrow f_{2}$. A much greater $\phi_{11 *}$ needs a smaller $\gamma_{1 *}$ to explain the intact covariances of $\left(x_{2}, x_{3}\right)$ with $\left(x_{4}, x_{5}, x_{6}\right)$. A greater $\phi_{11 *} \gamma_{1 *}$ also leads to a smaller $\varphi_{22} *$ for the intact $m_{f_{2} f_{2} *}$. Notice that $\sigma_{11}$ is positively perturbed by the additional $\lambda_{120}>0$. We are unable to predict the directions of change in $\psi_{11 *}$ to $\psi_{33 *}$.

When $x_{2}$ needs to load on $f_{2}$ with $\lambda_{220}>0, m_{x_{2} f_{2} *}=\lambda_{2 *} \phi_{11 *} \gamma_{1 *}$ has to be greater. Because $m_{f_{1} f_{2} *}=\phi_{11 *} \gamma_{1 *}$ is shared by all the between-cluster covariances, $\lambda_{2 *}$ has to be greater. There is no reason for $\phi_{11 *} \gamma_{1 *}$ to become smaller. A greater $\lambda_{2 *}$ needs a smaller $\phi_{11 *}$ to explain the covariances within $\left(x_{1}, x_{2}, x_{3}\right)$. A smaller $\phi_{11 *}$ leads to a greater $\psi_{11 *}$. A smaller $\phi_{11 *}$ also needs a greater $\gamma_{1 *}$ for not a smaller $\phi_{11 *} \gamma_{1 *}$. Because $\phi_{11 *} \gamma_{1 *}$ cannot be smaller, $\varphi_{22} *$ is most likely smaller. We are unable to predict the directions of change in $\lambda_{3 *}$, $\psi_{22 *}$, and $\psi_{33 *}$.

When $x_{4}$ needs to load on $f_{1}$ with $\lambda_{410}>0, m_{x_{4} f_{1} *}=\phi_{110} \gamma_{1 *}$ has to be greater, thus $\gamma_{1 *}$ has to be greater. A greater $\gamma_{1 *}$ needs smaller $\lambda_{5 *}$ and $\lambda_{6 *}$ to explain the intact $\sigma_{x_{5} f_{1}}$ and $\sigma_{x_{6} f_{1}}$. Smaller $\lambda_{5 *}$ and $\lambda_{6 *}$ need a greater $m_{f_{2} f_{2} *}$ to interpret the intact $\sigma_{56}$. Because $\sigma_{44}, \sigma_{45}$, and $\sigma_{46}$ are positively perturbed by $\lambda_{410}>0$, we are unable to predict the directions of change in $\varphi_{22 *}$ and $\psi_{44 *}$ to $\psi_{66 *}$.

When $x_{5}$ needs to load on $f_{1}$ with $\lambda_{510}>0, m_{x_{5} f_{1} *}=\lambda_{5 *} \phi_{11 *} \gamma_{1 *}$ has to be greater. Because $m_{f_{1} f_{2} *}=\phi_{11 *} \gamma_{1 *}$ is shared by all the between-cluster covariances, $\lambda_{5 *}$ has to be much greater. There is no reason for $\phi_{11 *} \gamma_{1 *}$ to become smaller and $\gamma_{1 *}$ also needs to be greater. A greater $\phi_{11 *} \gamma_{1 *}$ needs a smaller $\lambda_{6 *}$ for the intact between-cluster covariances $\sigma_{16}, \sigma_{26}$, and $\sigma_{36}$. We are unable to predict the directions of change in $\varphi_{22 *}$ and $\psi_{44 *}$ to $\psi_{66 *}$.

\section{A Model With Three Factors}

Figure 6 is a path diagram for a structural equation model with nine indicators and three factors, where $m_{f_{1} f_{1}}=\phi_{11}, m_{d_{j} d_{j}}=\varphi_{j j}, j=2,3 ; m_{e_{i} e_{i}}=\psi_{i i}$, $i=1$ to 9 . In this model, $\lambda_{2}, \lambda_{3}$, and $\phi_{11}$ are responsible for explaining the covariances within the cluster $\left(x_{1}, x_{2}, x_{3}\right)$. They are also involved in the between-cluster covariances among the indicators. Similarly, $\lambda_{5}, \lambda_{6}$, and $m_{f_{2} f_{2}}$ are mainly responsible for explaining the covariances within $\left(x_{4}, x_{5}, x_{6}\right) ; \lambda_{8}$, $\lambda_{9}$, and $m_{f_{3} f_{3}}$ are mainly responsible for explaining the covariances within $\left(x_{7}, x_{8}, x_{9}\right)$. The parameters $\gamma_{1}$ to $\gamma_{3}$ need to explain the relation between the clusters. The $\psi_{i i}$ s are mainly responsible for explaining the marginal variances of the observed variables. Due to the three predicted relations among the latent factors in Figure 6, analyzing the path is relatively more complicated than that for the one predicted relation in Figure 5. We need to use covariance algebra to facilitate the analysis. Let $\mathbf{f}=\left(f_{1}, f_{2}, f_{3}\right)^{\prime}$. The Appendix provides the detail 


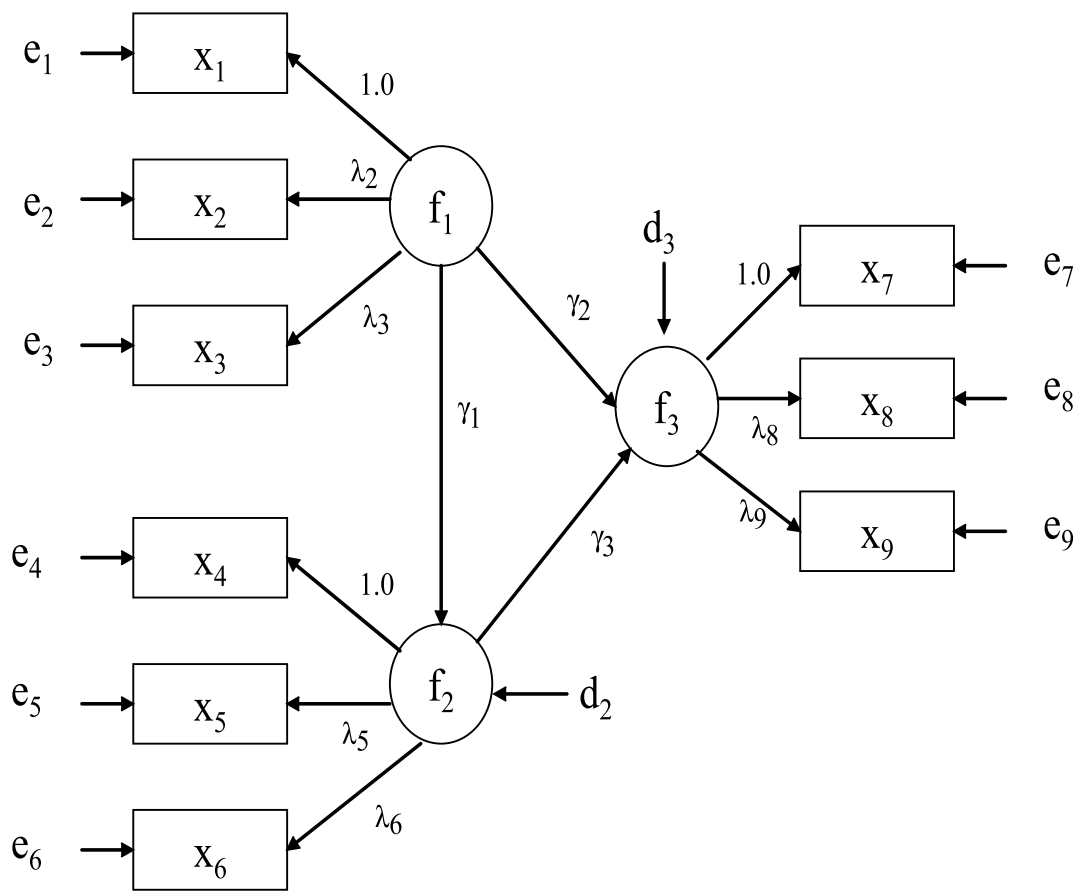

FIGURE 6 The path diagram for a structural equation model with three factors.

leading to the model-implied covariance matrix

$$
\operatorname{Cov}(\mathbf{f})=\left(\begin{array}{cc}
\phi_{11} & \gamma_{1} \phi_{11} \\
\gamma_{1} \phi_{11} & \gamma_{1}^{2} \phi_{11}+\varphi_{22} \\
\phi_{11}\left(\gamma_{2}+\gamma_{1} \gamma_{3}\right) & \gamma_{1} \phi_{11}\left(\gamma_{2}+\gamma_{1} \gamma_{3}\right)+\gamma_{3} \varphi_{22} \\
\phi_{11}\left(\gamma_{2}+\gamma_{1} \gamma_{3}\right) \\
\gamma_{1} \phi_{11}\left(\gamma_{2}+\gamma_{1} \gamma_{3}\right)+\gamma_{3} \varphi_{22} \\
\phi_{11}\left(\gamma_{2}+\gamma_{1} \gamma_{3}\right)^{2}+\gamma_{3}^{2} \varphi_{22}+\varphi_{33}
\end{array}\right) .
$$

The model-implied covariance between any two indicators is the two factor loadings times the corresponding element in this matrix. Due to $f_{1} \rightarrow f_{3} \leftarrow f_{2}$, some of the parameters in Figure 6 need to play different roles from the parallel ones in Figure 5. For example, the only duty of $\varphi_{22 *}$ in Figure 5 is to adjust for $m_{f_{2} f_{2} *}$; any changes in $\phi_{11 *}$ and $\gamma_{1 *}$ do not need to consider $m_{f_{2} f_{2} *}$. In 
Figure 6, $\varphi_{22}$ is also involved in $m_{f_{2} f_{3}}$ and $m_{f_{3} f_{3}}$. When a parameter is involved in more model-implied variances and covariances, it will be subjected to more restraints, but the principle of the analysis will remain the same. With a greater population covariance between two variables, the direct or shortest path between the two will mainly respond to the misspecification. Other paths will also be affected, but to a lesser degree. We first discuss the directions of change for the model parameters when errors are correlated, followed by the effect of double factor loadings in the population but not in the model, and then that of excluding the $\gamma \mathrm{s}$. When considering double factor loadings represented by paths $x_{1} \leftarrow f_{2}, x_{1} \leftarrow f_{3}, x_{2} \leftarrow f_{2}, x_{2} \leftarrow f_{3}, x_{4} \leftarrow f_{1}, x_{4} \leftarrow f_{3}, x_{5} \leftarrow f_{1}$, $x_{5} \leftarrow f_{3}, x_{7} \leftarrow f_{1}, x_{7} \leftarrow f_{2}, x_{8} \leftarrow f_{1}, x_{8} \leftarrow f_{2}$ we will use, respectively, $\lambda_{12}, \lambda_{13}, \lambda_{22}, \lambda_{23}, \lambda_{41}, \lambda_{43}, \lambda_{51}, \lambda_{53}, \lambda_{71}, \lambda_{72}, \lambda_{81}$, and $\lambda_{82}$ to denote the loading parameters.

Within the cluster $\left(x_{1}, x_{2}, x_{3}\right)$. Suppose a pair of errors within the cluster $\left(x_{1}, x_{2}, x_{3}\right)$ are positively correlated in Figure 6 . Similar to our analysis for the confirmatory three-factor model in the previous section and the two-factor structural equation model in this section, the within-cluster covariances of $\left(x_{4}, x_{5}, x_{6}\right)$ and $\left(x_{7}, x_{8}, x_{9}\right)$ are not affected, nor are the between-cluster associations of $\left(x_{4}, x_{5}, x_{6}\right)$ with $\left(x_{7}, x_{8}, x_{9}\right)$. Parameters $\lambda_{5}, \lambda_{6}, \lambda_{8}, \lambda_{9}$, and $\psi_{44}$ to $\psi_{99}$ will be correctly evaluated. The model-implied variances and covariance $m_{f_{2} f_{2}}, m_{f_{2} f_{3}}$, and $m_{f_{3} f_{3}}$ will also equal their population counterparts corresponding to the correctly specified model. The remaining analysis for excluding within-cluster error covariances is also similar to that in obtaining Tables 1 and 2. The results are summarized in Table 3. Readers are strongly encouraged to analyze Figure 6 themselves and compare their results with those in Table 3. Those who cannot get the same results may consult the following paragraphs corresponding to the exclusion of each error covariance.

When $\sigma_{e_{1} e_{2}}>0$, the greater $\sigma_{12}$ has to be explained through the path $x_{1} \leftarrow$ $f_{1} \rightarrow x_{2}$ by the model-implied covariance $m_{12}=\lambda_{2} \phi_{11}$. Thus, $\lambda_{2 *} \phi_{11 *}>$ $\lambda_{20} \phi_{110}$. Because $m_{23}=\lambda_{3} m_{12}$, there exists $\lambda_{3 *}<\lambda_{30}$. Notice that the positions of $\lambda_{2}$ and $\lambda_{3}$ are parallel in Figure 6, similar to that in Figure 5, a greater $\phi_{11 *}$ alone takes care of the extra association between $x_{1}$ and $x_{2}$. Actually, if $\lambda_{2 *}$ becomes greater and $\lambda_{3 *}$ becomes smaller, the model cannot explain the associations of $x_{2}$ and $x_{3}$ with the other two clusters. Due to a greater $\phi_{11 *}, \psi_{11 *}$ becomes smaller. To explain the extra covariance in $\sigma_{12}$, the average association of $x_{1}, x_{2}$, and $x_{3}$ with $f_{1}$, given by $\left(m_{x_{1} f_{1} *}+m_{x_{2} f_{1} *}+m_{x_{3} f_{1} *}\right)=\phi_{11 *}(1+$ $\left.\lambda_{2 *}+\lambda_{3 *}\right) / 3$, is stronger. The parameters $\gamma_{1}$ and $\gamma_{2}$ need to explain the betweencluster covariances of $\left(x_{1}, x_{2}, x_{3}\right)$ through $f_{1}$ with $\left(x_{4}, x_{5}, x_{6}\right)$ and $\left(x_{7}, x_{8}, x_{9}\right)$. They have to be smaller to explain the intact between-cluster covariances in the population. We cannot determine the directions of change in $\gamma_{3 *}, \varphi_{22 *}, \varphi_{33 *}$, $\psi_{22 *}$. and $\psi_{33 *}$. 
TABLE 3

The Directions of Change in Parameters When a Within-Cluster Positive Error Covariance Exists in the Population and the Model is Represented by Figure 6

\begin{tabular}{ccccccc}
\hline$\theta_{*}$ & $\sigma_{e_{1} e_{2}}$ & $\sigma_{e_{2} e_{3}}$ & $\sigma_{e_{4} e_{5}}$ & $\sigma_{e_{5} e_{6}}$ & $\sigma_{e_{7} e_{8}}$ & $\sigma_{e_{8} e_{9}}$ \\
\hline$\lambda_{2 *}$ & - & + & 0 & 0 & 0 & 0 \\
$\lambda_{3 *}$ & - & + & 0 & 0 & 0 & 0 \\
$\lambda_{5 *}$ & 0 & 0 & - & + & 0 & 0 \\
$\lambda_{6 *}$ & 0 & 0 & - & + & 0 & 0 \\
$\lambda_{8 *}$ & 0 & 0 & 0 & 0 & - & + \\
$\lambda_{9 *}$ & 0 & 0 & 0 & 0 & - & + \\
$\phi_{11 *}$ & + & - & 0 & 0 & 0 & 0 \\
$\gamma_{1 *}$ & - & - & + & - & 0 & 0 \\
$\gamma_{2 *}$ & - & - & $?$ & $?$ & + & - \\
$\gamma_{3 *}$ & $?$ & $?$ & $?$ & $?$ & + & - \\
$\varphi_{22 *}$ & $?$ & + & + & $?$ & 0 & 0 \\
$\varphi_{33 *}$ & $?$ & $?$ & $?$ & $?$ & + & $?$ \\
$\psi_{11 *}$ & - & + & 0 & 0 & 0 & 0 \\
$\psi_{22 *}$ & $?$ & $?$ & 0 & 0 & 0 & 0 \\
$\psi_{33 *}$ & $?$ & $?$ & 0 & 0 & 0 & 0 \\
$\psi_{44 *}$ & 0 & 0 & - & + & 0 & 0 \\
$\psi_{55 *}$ & 0 & 0 & $?$ & $?$ & 0 & 0 \\
$\psi_{66 *}$ & 0 & 0 & $?$ & $?$ & 0 & 0 \\
$\psi_{77 *}$ & 0 & 0 & 0 & 0 & - & + \\
$\psi_{88 *}$ & 0 & 0 & 0 & 0 & $?$ \\
$\psi_{99 *}$ & 0 & 0 & 0 & 0 & $?$ \\
\hline & & & 0 & & $?$ \\
\hline
\end{tabular}

When $\sigma_{e_{2} e_{3}}>0, m_{23 *}=\lambda_{2 *} \lambda_{3 *} \phi_{11 *}$ has to be greater. It is impossible for $\lambda_{2 *}$ or $\lambda_{3 *}$ to be smaller. Due to greater $\lambda_{2 *}$ and $\lambda_{3 *}, \phi_{11 *}$ has to be smaller. For the same reason as when $\sigma_{e_{1} e_{2}}>0, \gamma_{1 *}$ and $\gamma_{2 *}$ have to be smaller. A smaller $\phi_{11 *}$ together with a smaller $\gamma_{1 *}$ leads to a greater $\varphi_{22 *}$. A smaller $\phi_{11 *}$ leads to a greater $\psi_{11 *}$. We are unable to determine the directions of change in $\gamma_{3 *}$, $\varphi_{33 *}, \psi_{22 *}$, and $\psi_{33 *}$.

Within the cluster $\left(x_{4}, x_{5}, x_{6}\right)$. As summarized in Table 3 , the misspecification of ignoring error covariances within $\left(x_{4}, x_{5}, x_{6}\right)$ does not affect the evaluation of the within-cluster parameters of $\left(x_{1}, x_{2}, x_{3}\right)$ and $\left(x_{7}, x_{8}, x_{9}\right)$. The variances and covariance $m_{f_{1} f_{1} *}=\phi_{11 *}, m_{f_{1} f_{3} *}=\phi_{11 *}\left(\gamma_{2 *}+\gamma_{1 *} \gamma_{3 *}\right)$, and $m_{f_{3} f_{3} *}=\phi_{11 *}\left(\gamma_{2 *}+\gamma_{1 *} \gamma_{3 *}\right)^{2}+\gamma_{3 *}^{2} \varphi_{22 *}+\varphi_{33 *}$ will also equal their population counterparts corresponding to the correctly specified model.

When $\sigma_{e_{4} e_{5}}>0, \sigma_{45}$ will be greater, which has to be explained by $m_{45}=$ $\lambda_{5} m_{f_{2} f_{2}}$. For the same reason as in Figure $5, m_{f_{2} f_{2} *}$ will be greater, $\lambda_{5 *}$ and $\lambda_{6 *}$ will be smaller, and $\psi_{44 *}$ will be smaller. Due to smaller $\lambda_{5 *}$ and $\lambda_{6 *}$, and 
$m_{f_{1} f_{2} *}=\gamma_{1 *} \phi_{110}, \gamma_{1 *}$ has to be greater to explain the intact between-cluster covariances of $\left(x_{1}, x_{2}, x_{3}\right)$ with $\left(x_{4}, x_{5}, x_{6}\right)$. Notice that $m_{f_{2} f_{2}}=\gamma_{1}^{2} \phi_{11}+\varphi_{22}$. Because $\gamma_{1}$ is mainly responsible for the between-cluster associations, a greater $\gamma_{1 *}$ alone is not enough for the greater $\sigma_{45}$, and $\varphi_{22} *$ should be greater for the greater $m_{f_{2} f_{2} *}$. We are unable to determine the directions of change in $\gamma_{2 *}, \gamma_{3 *}$, $\varphi_{33 *}, \psi_{55 *}$, and $\psi_{66 *}$.

When $\sigma_{e_{5} e_{6}}>0$, similar to when $\sigma_{e_{2} e_{3}}>0, \lambda_{5 *}$ and $\lambda_{6 *}$ will be greater, $m_{f_{2} f_{2} *}$ has to be smaller. Notice that $m_{f_{1} f_{2} *}=\gamma_{1 *} \phi_{110}$. Due to greater $\lambda_{5 *}$ and $\lambda_{6 *}, \gamma_{1 *}$ has to be smaller to explain the between-cluster covariances of $\left(x_{1}, x_{2}, x_{3}\right)$ with $\left(x_{4}, x_{5}, x_{6}\right)$. A smaller $m_{f_{2} f_{2} *}$ leads to a greater $\psi_{44 *}$. Due to the stronger associations within $\left(x_{4}, x_{5}, x_{6}\right), \gamma_{3 *}$ will change to adjust for the between-cluster covariances. Because too many parameters affect $m_{f_{2} f_{3}}$, we are unable to determine the directions of change in $\gamma_{3 *}$. We are unable to determine the directions of change in $\gamma_{2 *}, \varphi_{22 *}, \varphi_{33 *}, \psi_{55 *}$, and $\psi_{66 *}$ either.

Within the cluster $\left(x_{7}, x_{8}, x_{9}\right)$. Using the same logic as in the previous sections, ignoring possible covariances among $e_{7}, e_{8}$, and $e_{9}$ does not affect the parameters $\lambda_{2}, \lambda_{3}, \lambda_{5}, \lambda_{6}$, and $\psi_{11}$ to $\psi_{66}$. Because $m_{f_{1} f_{1}}, m_{f_{1} f_{2}}$, and $m_{f_{2} f_{2}}$ are also correctly evaluated, $\phi_{11}, \gamma_{1}$, and $\varphi_{22}$ will not be affected either.

When $\sigma_{e_{7} e_{8}}>0, m_{f_{3} f_{3} *}$ is greater, $\lambda_{8 *}$ and $\lambda_{9 *}$ are smaller, and $\psi_{77 *}$ is smaller. Because $m_{f_{3} f_{3}}=\phi_{11}\left(\gamma_{2}+\gamma_{1} \gamma_{3}\right)^{2}+\gamma_{3}^{2} \varphi_{22}+\varphi_{33}$ and $\gamma_{2}$ and $\gamma_{3}$ are mainly responsible for the between-cluster associations, the only function of $\varphi_{33}$ is to adjust for $m_{f_{3} f_{3}}$; thus, $\varphi_{33 *}$ should be greater. Note that $\varphi_{33 *}$ does not contribute to the between-cluster associations. Due to smaller $\lambda_{8 *}$ and $\lambda_{9 *}, \gamma_{2 *}$ and $\gamma_{3 *}$ need to be greater to explain the intact between-cluster covariances of $\left(x_{7}, x_{8}, x_{9}\right)$ with $\left(x_{1}, x_{2}, x_{3}\right)$ and $\left(x_{4}, x_{5}, x_{6}\right)$. We are unable to determine the directions of change in $\psi_{88 *}$ and $\psi_{99 *}$.

When $\sigma_{e_{8} e_{9}}>0$, both $\lambda_{8 *}$ and $\lambda_{9 *}$ are greater, $m_{f_{3} f_{3} *}$ is smaller, and $\psi_{77 *}$ is greater. Due to the greater average path $\left(m_{x_{7} f_{3} *}+m_{x_{8} f_{3} *}+m_{x_{9} f_{3} *}\right) / 3, \gamma_{2 *}, \gamma_{3 *}$ will be smaller to explain the intact between-cluster covariances. We are unable to predict the directions of change in $\varphi_{33 *}, \psi_{88 *}$, and $\psi_{99 *}$.

Between clusters $\left(x_{1}, x_{2}, x_{3}\right)$ and $\left(x_{4}, x_{5}, x_{6}\right)$. First, the misspecification of ignoring a covariance between $\left(e_{1}, e_{2}, e_{3}\right)$ and $\left(e_{4}, e_{5}, e_{6}\right)$ does not affect the estimation of parameters within cluster $\left(x_{7}, x_{8}, x_{9}\right)$. Thus, $\lambda_{8 *}, \lambda_{9 *}, \psi_{77 *}, \psi_{88 *}$, and $\psi_{99 *}$ will equal their counterparts corresponding to the correctly specified model; $\sigma_{f_{3} f_{3}}$ will also be correctly predicted by $m_{f_{3} f_{3} *}$. The results for excluding between-cluster error covariances are summarized in Table 4. We encourage readers to analyze Figure 6 to obtain their own version of the table.

When $\sigma_{e_{1} e_{4}}>0$ in Figure 6, the path $f_{1} \rightarrow f_{2}$ is responsible for explaining the greater $\sigma_{14}$ with $m_{14}=m_{f_{1} f_{2}}=\gamma_{1} \phi_{11}$. Different from the model in Figure 5, 
TABLE 4

The Directions of Change in Parameters When a Between-Cluster Positive Error Covariance Exists in the Population and the Model is Represented by Figure 6

\begin{tabular}{ccccccccccccc}
\hline$\theta$ & $\sigma_{e_{1} e_{4}}$ & $\sigma_{e_{1} e_{5}}$ & $\sigma_{e_{2} e_{4}}$ & $\sigma_{e_{2} e_{5}}$ & $\sigma_{e_{1} e_{7}}$ & $\sigma_{e_{1} e_{8}}$ & $\sigma_{e_{2} e_{7}}$ & $\sigma_{e_{2} e_{8}}$ & $\sigma_{e_{4} e_{7}}$ & $\sigma_{e_{4} e_{8}}$ & $\sigma_{e_{5} e_{7}}$ & $\sigma_{e_{5} e_{8}}$ \\
\hline$\lambda_{2 *}$ & $?$ & $?$ & $?$ & $?$ & - & - & + & + & 0 & 0 & 0 & 0 \\
$\lambda_{3 *}$ & $?$ & $?$ & $?$ & $?$ & - & - & - & - & 0 & 0 & 0 & 0 \\
$\lambda_{5 *}$ & $?$ & $?$ & $?$ & $?$ & 0 & 0 & 0 & 0 & - & - & + & + \\
$\lambda_{6 *}$ & $?$ & $?$ & $?$ & $?$ & 0 & 0 & 0 & 0 & - & - & - & - \\
$\lambda_{8 *}$ & 0 & 0 & 0 & 0 & - & + & - & + & - & + & - & + \\
$\lambda_{9 *}$ & 0 & 0 & 0 & 0 & - & - & - & - & - & - & - & - \\
$\phi_{11 *}$ & $?$ & $?$ & $?$ & $?$ & + & + & - & - & 0 & 0 & 0 & 0 \\
$\gamma_{1 *}$ & + & + & + & + & - & - & + & + & + & + & $?$ & $?$ \\
$\gamma_{2 *}$ & $?$ & $?$ & $?$ & $?$ & + & + & + & + & - & - & $?$ & $?$ \\
$\gamma_{3 *}$ & $?$ & $?$ & $?$ & $?$ & $?$ & $?$ & $?$ & $?$ & + & + & + & + \\
$\varphi_{22 *}$ & - & - & - & - & $?$ & $?$ & $?$ & $?$ & + & + & $?$ & $?$ \\
$\varphi_{33 *}$ & $?$ & $?$ & $?$ & $?$ & $?$ & $?$ & $?$ & $?$ & $?$ & $?$ & $?$ & $?$ \\
$\psi_{11 *}$ & $?$ & $?$ & $?$ & $?$ & - & - & + & + & 0 & 0 & 0 & 0 \\
$\psi_{22 *}$ & $?$ & $?$ & $?$ & $?$ & $?$ & $?$ & $?$ & $?$ & 0 & 0 & 0 & 0 \\
$\psi_{33 *}$ & $?$ & $?$ & $?$ & $?$ & $?$ & $?$ & $?$ & $?$ & 0 & 0 & 0 & 0 \\
$\psi_{44 *}$ & $?$ & $?$ & $?$ & $?$ & 0 & 0 & 0 & 0 & - & - & $?$ & $?$ \\
$\psi_{55 *}$ & $?$ & $?$ & $?$ & $?$ & 0 & 0 & 0 & 0 & $?$ & $?$ & $?$ & $?$ \\
$\psi_{66 *}$ & $?$ & $?$ & $?$ & $?$ & 0 & 0 & 0 & 0 & $?$ & $?$ & $?$ & $?$ \\
$\psi_{77 *}$ & 0 & 0 & 0 & 0 & - & $?$ & $?$ & $?$ & - & $?$ & - & $?$ \\
$\psi_{88 *}$ & 0 & 0 & 0 & 0 & $?$ & $?$ & $?$ & $?$ & $?$ & $?$ & $?$ & $?$ \\
$\psi_{99 *}$ & 0 & 0 & 0 & 0 & $?$ & $?$ & $?$ & $?$ & $?$ & $?$ & $?$ & $?$ \\
\hline & & & & & & & & & & & & \\
\hline
\end{tabular}

$f_{1}$ is also at the origin of the prediction relation $f_{1} \rightarrow f_{3}$, the parameter $\phi_{11}$ is subject to the constraints of the intact covariances between $\left(x_{1}, x_{2}, x_{3}\right)$ and $\left(x_{7}, x_{8}, x_{9}\right)$. Because $m_{f_{3} f_{3}}$ is not affected by $\sigma_{e_{1} e_{4}}>0$, there is little flexibility in $\phi_{11}$. Although $\gamma_{1}$ also contributes to $m_{f_{3} f_{3}}$, the contribution is an indirect effect, so there is more flexibility in $\gamma_{1}$ than in $\phi_{11}$. Actually, $\phi_{11}$ has two contributions to $m_{f_{3} f_{3}}$; one is the direct effect through $f_{1} \rightarrow f_{3}$, and the other is the indirect effect through $f_{1} \rightarrow f_{2} \rightarrow f_{3}$. In the indirect effect, $\phi_{11}$ and $\gamma_{1}$ are comparable in their contributions. Thus, a greater $\gamma_{1 *} \phi_{11 *}$ needs a greater $\gamma_{1 *}$. With a greater $\gamma_{1 *}, \phi_{11 *}$ can be greater or smaller; a greater $\phi_{11 *}$ corresponds to smaller $\lambda_{2 *}$ and $\lambda_{3 *}$ and vice versa. However, it is hard to predict the direction of change in $\phi_{11 *}$. Similarly, $\lambda_{2 *}, \lambda_{3 *}, \lambda_{5 *}$ and $\lambda_{6 *}$ need to explain both the within- and between-cluster associations of $\left(x_{1}, x_{2}, x_{3}\right)$ and $\left(x_{4}, x_{5}, x_{6}\right)$. For example, $\sigma_{24}$ and $\sigma_{34}$ need smaller $\lambda_{2 *}$ and $\lambda_{3 *}$ whereas $\sigma_{12}, \sigma_{13}$, and $\sigma_{23}$ may need them to be greater. Consequently, it is hard to predict their directions of change. The parameter $\varphi_{22}$ mainly reflects the prediction error in $f_{1} \rightarrow f_{2} \leftarrow d_{2}$, a greater $\gamma_{1 *}$ will lead to a smaller $\varphi_{22 *}$. We are also unable to determine the directions of change in $\gamma_{2 *}, \gamma_{3 *}, \varphi_{33 *}$, and $\psi_{11 *}$ to $\psi_{66 *}$. 
Similar to $\sigma_{e_{1} e_{4}}>0$, when a positive covariance between one of $\left(e_{1}, e_{2}, e_{3}\right)$ and one of $\left(e_{4}, e_{5}, e_{6}\right)$ exists, $\gamma_{1 *}$ will be greater and $\varphi_{22 *}$ will be smaller. We are unable to determine the directions of change in $\lambda_{2 *}, \lambda_{3 *}, \lambda_{5 *}, \lambda_{6 *}, \gamma_{2 *}, \gamma_{3 *}$, and $\varphi_{33 *}$.

Between clusters $\left(x_{1}, x_{2}, x_{3}\right)$ and $\left(x_{7}, x_{8}, x_{9}\right)$. When ignoring a covariance between $\left(e_{1}, e_{2}, e_{3}\right)$ and $\left(e_{7}, e_{8}, e_{9}\right)$, the parameters within the cluster $\left(x_{4}, x_{5}, x_{6}\right)$ are not affected, as indicated in Table $4 ; m_{f_{2} f_{2} *}$ will be correctly evaluated. The shortest path from $x_{i}, i=1,2,3$ to $x_{j}, j=7,8,9$ is through $f_{1} \rightarrow f_{3}$. Although the indirect path $f_{1} \rightarrow f_{2} \rightarrow f_{3}$ may partially explain the covariances between $\left(x_{1}, x_{2}, x_{3}\right)$ and $\left(x_{7}, x_{8}, x_{9}\right)$, because the model parameters within the cluster $\left(x_{4}, x_{5}, x_{6}\right)$ are not affected, any extra association between $\left(x_{1}, x_{2}, x_{3}\right)$ and $\left(x_{7}, x_{8}, x_{9}\right)$ passed through the indirect path is very limited. A greater $\phi_{11 *}$ needs to pass both the direct and indirect paths to achieve a greater $m_{f_{1} f_{3} *}$, so $\gamma_{2 *}$ is mainly responsible for a greater $\sigma_{i j}, i=1,2,3$ and $j=7$, 8,9 . Notice that, unlike $\varphi_{22 *}$, the only function of $\varphi_{33 *}$ is to adjust for $m_{f_{3} f_{3} *}$; the covariances within the cluster $\left(x_{7}, x_{8}, x_{9}\right)$ have little to do with the values of $\gamma_{2 *}$ and $\phi_{11 *}$.

When $\sigma_{e_{1} e_{7}}>0, \gamma_{2 *}$ should be greater and so is $m_{17 *}=m_{f_{1} f_{3} *}$. Notice $m_{27}=\lambda_{2} m_{f_{1} f_{3}}$. With a greater $m_{f_{1} f_{3} *}, \lambda_{2 *}$ has to be smaller to explain $\sigma_{27}$. Similarly, $\lambda_{3 *}, \lambda_{8 *}$, and $\lambda_{9 *}$ have to be smaller. Due to smaller $\lambda_{2 *}$ and $\lambda_{3 *}$, $\phi_{11 *}$ has to be greater to explain the within-cluster covariances of $\left(x_{1}, x_{2}, x_{3}\right)$. A greater $\phi_{11 *}$ implies smaller $\gamma_{1 *}$ and $\psi_{11 *}$. Similarly, $m_{f_{3} f_{3} *}$ has to be greater and $\psi_{77 *}$ has to be smaller. Our analysis cannot determine the directions of change in $\gamma_{3 *}, \varphi_{22 *}, \varphi_{33 *}, \psi_{22 *}, \psi_{33 *}, \psi_{88 *}$, and $\psi_{99 *}$.

When $\sigma_{e_{1} e_{8}}>0, \gamma_{2 *}$ should be greater and so are $m_{f_{1} f_{3} *}$ and $m_{18 *}$. Because $m_{18}=\lambda_{8} m_{f_{1} f_{3}}$ and $m_{f_{1} f_{3}}$ is shared by all the paths between $\left(x_{1}, x_{2}, x_{3}\right)$ and $\left(x_{7}, x_{8}, x_{9}\right), \lambda_{8 *}$ has to be greater. Notice $m_{28}=\lambda_{2} m_{18}, m_{38}=\lambda_{3} m_{18}$, and $m_{19}=\lambda_{9} m_{f_{1} f_{3}}$; hence $\lambda_{2 *}, \lambda_{3 *}$, and $\lambda_{9 *}$ have to be smaller. Due to smaller $\lambda_{2 *}$ and $\lambda_{3 *}, \phi_{11 *}$ has to be greater to explain the within-cluster associations of $\left(x_{1}, x_{2}, x_{3}\right)$. A greater $\phi_{11 *}$ leads to smaller $\gamma_{1 *}$ and $\psi_{11 *}$. We are unable to determine the directions of change in $\gamma_{3 *}, \varphi_{22 *}, \varphi_{33 *}, \psi_{22 *}, \psi_{33 *}$, and $\psi_{77 *}$ to $\psi_{99 *}$.

When $\sigma_{e_{2} e_{7}}>0$, the direct path loading $\gamma_{2 *}$ from $f_{1}$ to $f_{3}$ as well as $m_{f_{1} f_{3} *}$, $m_{27} *$ and $\lambda_{2 *}$ should be greater. Notice $m_{28}=\lambda_{8} m_{27}$ and $m_{29}=\lambda_{9} m_{27}, m_{37}=$ $\lambda_{3} m_{f_{1} f_{3}}$; hence $\lambda_{3 *}, \lambda_{8 *}$, and $\lambda_{9 *}$ have to be smaller for the corresponding intact between-cluster covariances. Due to greater $\lambda_{2 *}, \phi_{11 *}$ should be smaller to explain $\sigma_{12}$. A smaller $\phi_{11 *}$ leads to greater $\gamma_{1 *}$ and $\psi_{11 *}$. Our analysis cannot determine the directions of change in $\gamma_{3 *}, \varphi_{22 *}, \varphi_{33 *}, \psi_{22 *}, \psi_{33 *}$, and $\psi_{77 *}$ to $\psi_{99 *}$.

When $\sigma_{e_{2} e_{8}}>0$, both $\lambda_{2 *}$ and $\lambda_{8 *}$ should be greater. Due to a greater $\lambda_{2 *}$ and $\lambda_{8 *}, \phi_{11 *}, \lambda_{3 *}$, and $\lambda_{9 *}$ have to be smaller to explain the intact within-cluster covariances. A smaller $\phi_{11 *}$ needs a greater $\gamma_{2 *}$; a smaller $\phi_{11 *}$ also leads to a 
greater $\gamma_{1 *}$ and $\psi_{11 *}$. Our analysis cannot determine the directions of change in $\gamma_{3 *}, \varphi_{22 *}, \varphi_{33 *}, \psi_{22 *}, \psi_{33 *}$, and $\psi_{77 *}$ to $\psi_{99 *}$.

Between clusters $\left(x_{4}, x_{5}, x_{6}\right)$ and $\left(x_{7}, x_{8}, x_{9}\right)$. With possible covariances between $\left(e_{4}, e_{5}, e_{6}\right)$ and $\left(e_{7}, e_{8}, e_{9}\right)$ in Figure 6 , the parameters within the cluster $\left(x_{1}, x_{2}, x_{3}\right)$ are not affected, nor is $\phi_{11}=m_{f_{1} f_{1}}$. The path $f_{2} \rightarrow f_{3}$ is mainly responsible for explaining the covariances between the indicators in $\left(x_{4}, x_{5}, x_{6}\right)$ and $\left(x_{7}, x_{8}, x_{9}\right)$. Although $m_{f_{2} f_{3}}$ involves $\gamma_{1}$ and $\gamma_{2}$, due to $\phi_{11 *}=\phi_{110}$ and the intact between-cluster covariances of $\left(x_{1}, x_{2}, x_{3}\right)$ with $\left(x_{4}, x_{5}, x_{6}\right)$ and $\left(x_{7}, x_{8}, x_{9}\right)$, the strength of $\gamma_{1 *}$ and $\gamma_{2 *}$ to affect $m_{f_{2} f_{3}}$ is limited. Actually, $\gamma_{1}$ is mainly responsible for the between-cluster associations of $\left(x_{1}, x_{2}, x_{3}\right)$ and $\left(x_{4}, x_{5}, x_{6}\right) ; \gamma_{2}$ is mainly responsible for the between-cluster associations of $\left(x_{1}, x_{2}, x_{3}\right)$ and $\left(x_{7}, x_{8}, x_{9}\right)$.

When $\sigma_{e_{4} e_{7}}>0$, both $\gamma_{3 *}$ and $m_{47}=m_{f_{2} f_{3} *}$ have to be greater. Notice $m_{57}=\lambda_{5} m_{47}, m_{67}=\lambda_{6} m_{47}, m_{48}=\lambda_{8} m_{47}$, and $m_{49}=\lambda_{9} m_{47}$. Due to a greater $m_{47 *}, \lambda_{5 *}, \lambda_{6 *}, \lambda_{8 *}$, and $\lambda_{9 *}$ have to be smaller. Due to smaller $\lambda_{5 *}$ and $\lambda_{6 *}$, $m_{f_{2} f_{2} *}$ has to be greater to explain the within-cluster covariances of $\left(x_{4}, x_{5}, x_{6}\right)$; $\psi_{44 *}$ has to be smaller. The parameter $\varphi_{22 *}$ should be greater because it is mainly responsible for adjusting the variance of $f_{2}$. Due to the smaller average loadings within the cluster $\left(x_{4}, x_{5}, x_{6}\right), \gamma_{1 *}$ has to be greater to explain the between-cluster associations of $\left(x_{1}, x_{2}, x_{3}\right)$ with $\left(x_{4}, x_{5}, x_{6}\right)$. Notice $m_{f_{1} f_{3}}=\phi_{11}\left(\gamma_{1} \gamma_{3}+\gamma_{2}\right)$. Due to greater $\gamma_{1 *}$ and $\gamma_{3 *}, \gamma_{2 *}$ has to be smaller. Due to smaller $\lambda_{8 *}$ and $\lambda_{9 *}, m_{f_{3} f_{3} *}$ is greater, and $\psi_{77 *}$ is smaller. We cannot determine the directions of change in $\varphi_{33 *}, \psi_{55 *}, \psi_{66 *}, \psi_{88 *}$, and $\psi_{99 *}$.

When $\sigma_{e_{4} e_{8}}>0, \gamma_{3 *}$ as well as $m_{48 *}$ and $m_{f_{2} f_{3} *}$ will be greater. Due to the unique position of $x_{8}, \lambda_{8 *}$ should be greater. Notice that $m_{49}=\lambda_{9} m_{f_{2} f_{3}}$, $m_{58}=\lambda_{5} m_{48}$, and $m_{68}=\lambda_{6} m_{48}$, hence $\lambda_{5 *}, \lambda_{6 *}$, and $\lambda_{9 *}$ have to be smaller. Due to smaller $\lambda_{5 *}$ and $\lambda_{6 *}, m_{f_{2} f_{2} *}$ has to be greater to explain the intact withincluster covariances of $\left(x_{4}, x_{5}, x_{6}\right)$. Consequently, $\varphi_{22 *}$, whose main function is to adjust for $m_{f_{2} f_{2} *}$, has to be greater; $\psi_{44 *}$ has to be smaller. Because $\gamma_{1}$ is mainly for the between-cluster associations of $\left(x_{1}, x_{2}, x_{3}\right)$ with $\left(x_{4}, x_{5}, x_{6}\right)$ and the average factor loading within the cluster $\left(x_{4}, x_{5}, x_{6}\right)$ is smaller, $\gamma_{1 *}$ has to be greater. Notice $m_{f_{1} f_{3}}=\phi_{11}\left(\gamma_{2}+\gamma_{1} \gamma_{3}\right)$. Due to greater $\gamma_{1 *}$ and $\gamma_{3 *}, \gamma_{2 *}$ has to be smaller. We cannot determine the directions of change in $\varphi_{33 *}, \psi_{55 *}$ to $\psi_{99 *}$.

When $\sigma_{e_{5} e_{7}}>0, \lambda_{5 *}, m_{f_{2} f_{3} *}, m_{57 *}$ will be greater. Notice $m_{x_{6} f_{2}}=\lambda_{6} m_{f_{2} f_{3} *}$, $m_{58}=\lambda_{8} m_{57}$, and $m_{59}=\lambda_{9} m_{57}$; hence $\lambda_{6 *}, \lambda_{8 *}$, and $\lambda_{9 *}$ need to be smaller. Due to smaller $\lambda_{8 *}$ and $\lambda_{9 *}, m_{f_{3} f_{3} *}$ will be greater, which leads to a smaller $\psi_{77 *}$. Our analysis cannot determine the directions of change in $\gamma_{1 *}, \gamma_{2 *}, \varphi_{22 *}$, $\varphi_{33 *}, \psi_{44 *}$ to $\psi_{66 *}, \psi_{88 *}$, and $\psi_{99 *}$.

When $\sigma_{e_{5} e_{8}}>0, \lambda_{5 *}, \lambda_{8 *}, m_{f_{2} f_{3} *}, m_{58 *}$ will be greater; $\lambda_{6 *}$ and $\lambda_{9 *}$ will be smaller. We are unable to determine the directions of change in $\gamma_{1 *}, \gamma_{2 *}, \varphi_{22 *}$, $\varphi_{33 *}, \psi_{44 *}$ to $\psi_{99 *}$. 
Extra factor loadings for $x_{1}$ or $x_{2}$. When $x_{1}$ or $x_{2}$ needs to load on $f_{2}$ or $f_{3}$, the model represented by Figure 6 is misspecified. Because the withinand between-cluster associations of $\left(x_{4}, x_{5}, x_{6}\right)$ and $\left(x_{7}, x_{8}, x_{9}\right)$ are not affected by the misspecification, $\lambda_{5 *}, \lambda_{6 *}, \lambda_{8 *}, \lambda_{9 *}$, and $\psi_{44 *}$ to $\psi_{99 *}$ will equal their counterparts in the correctly specified model. The variances and covariance $m_{f_{2} f_{2} *}, m_{f_{2} f_{3} *}$, and $m_{f_{3} f_{3} *}$ will also be correctly evaluated. In the following we only discuss the remaining parameters when an extra loading exists in the population for the model represented by Figure 6. The results corresponding to excluding all the factor loadings are summarized in Table 5. Readers should analyze Figure 6 to get their own version of the table.

When $x_{1}$ needs to load on $f_{2}$ with $\lambda_{120}>0, m_{x_{1} f_{2} *}=\phi_{11 *} \gamma_{1 *}$ has to be greater. A greater $\phi_{11 *} \gamma_{1 *}$ needs smaller $\lambda_{2 *}$ and $\lambda_{3 *}$ to explain the intact $\sigma_{x_{2} f_{2}}$ and $\sigma_{x_{3} f_{2}}$. Smaller $\lambda_{2 *}$ and $\lambda_{3 *}$ need a much greater $\phi_{11 *}$ to interpret the intact $\sigma_{23}$ and greater $\sigma_{12}$ and $\sigma_{13}$. A much greater $\phi_{11 *}$ needs a smaller $\gamma_{1 *}$ to explain the intact covariances of $\left(x_{2}, x_{3}\right)$ with $\left(x_{4}, x_{5}, x_{6}\right)$. For a similar reason, $\gamma_{2 *}$ needs to be smaller. A much greater $\phi_{11 *}$ or greater $\phi_{11 *} \gamma_{1 *}$ also leads to a smaller $\varphi_{22 *}$ to have $m_{f_{2} f_{2} *}=m_{f_{2} f_{2} 0}$. We are unable to determine the directions

TABLE 5

The Directions of Change in Parameters When an Extra Positive Factor Loading Exists in the Population and the Model is Represented by Figure 6

\begin{tabular}{ccccccccccccc}
\hline$\theta$ & $\lambda_{120}$ & $\lambda_{130}$ & $\lambda_{220}$ & $\lambda_{230}$ & $\lambda_{410}$ & $\lambda_{430}$ & $\lambda_{510}$ & $\lambda_{530}$ & $\lambda_{710}$ & $\lambda_{720}$ & $\lambda_{810}$ & $\lambda_{820}$ \\
\hline$\lambda_{2 *}$ & - & - & + & + & 0 & 0 & 0 & 0 & 0 & 0 & 0 & 0 \\
$\lambda_{3 *}$ & - & - & - & - & 0 & 0 & 0 & 0 & 0 & 0 & 0 & 0 \\
$\lambda_{5 *}$ & 0 & 0 & 0 & 0 & - & - & + & + & 0 & 0 & 0 & 0 \\
$\lambda_{6 *}$ & 0 & 0 & 0 & 0 & - & - & - & - & 0 & 0 & 0 & 0 \\
$\lambda_{8 *}$ & 0 & 0 & 0 & 0 & 0 & 0 & 0 & 0 & - & - & + & + \\
$\lambda_{9 *}$ & 0 & 0 & 0 & 0 & 0 & 0 & 0 & 0 & - & - & - & - \\
$\phi_{11 *}$ & + & + & - & - & 0 & 0 & 0 & 0 & 0 & 0 & 0 & 0 \\
$\gamma_{1 *}$ & - & - & + & + & + & + & + & $?$ & 0 & 0 & 0 & 0 \\
$\gamma_{2 *}$ & - & - & + & + & - & - & $?$ & $?$ & + & + & + & - \\
$\gamma_{3 *}$ & $?$ & $?$ & $?$ & $?$ & - & - & $?$ & + & $?$ & + & $?$ & + \\
$\varphi_{22 *}$ & - & - & $?$ & $?$ & $?$ & $?$ & - & $?$ & 0 & 0 & 0 & 0 \\
$\varphi_{33 *}$ & $?$ & $?$ & $?$ & $?$ & $?$ & $?$ & $?$ & $?$ & $?$ & $?$ & $?$ & $?$ \\
$\psi_{11 *}$ & $?$ & $?$ & + & + & 0 & 0 & 0 & 0 & 0 & 0 & 0 & 0 \\
$\psi_{22 *}$ & $?$ & $?$ & $?$ & $?$ & 0 & 0 & 0 & 0 & 0 & 0 & 0 & 0 \\
$\psi_{33 *}$ & $?$ & $?$ & + & + & 0 & 0 & 0 & 0 & 0 & 0 & 0 & 0 \\
$\psi_{44 *}$ & 0 & 0 & 0 & 0 & $?$ & $?$ & + & + & 0 & 0 & 0 & 0 \\
$\psi_{55 *}$ & 0 & 0 & 0 & 0 & $?$ & $?$ & $?$ & $?$ & 0 & 0 & 0 & 0 \\
$\psi_{66 *}$ & 0 & 0 & 0 & 0 & $?$ & $?$ & + & + & 0 & 0 & 0 & 0 \\
$\psi_{77 *}$ & 0 & 0 & 0 & 0 & 0 & 0 & 0 & 0 & $?$ & $?$ & + & + \\
$\psi_{88 *}$ & 0 & 0 & 0 & 0 & 0 & 0 & 0 & 0 & $?$ & $?$ & $?$ & $?$ \\
$\psi_{99 *}$ & 0 & 0 & 0 & 0 & 0 & 0 & 0 & 0 & $?$ & $?$ & + & + \\
\hline
\end{tabular}


of change in $\gamma_{3 *}$ and $\varphi_{33 *}$. Due to $\lambda_{120}>0$ positively perturbing $\sigma_{11}$, we are unable to determine the directions of change in $\psi_{11 *}$ to $\psi_{33 *}$ either.

When $x_{1}$ needs to load on $f_{3}$ with $\lambda_{130}>0$, the stronger association between $x_{1}$ and $f_{3}$ has to be explained by $m_{x_{1} f_{3}}$. By similar logic as with $\lambda_{120}>0, \phi_{11 *}$ has to be much greater; $\lambda_{2 *}$ and $\lambda_{3 *}$ have to be smaller; and $\gamma_{1 *}, \gamma_{2 *}$, and $\varphi_{22 *}$ have to be smaller. We are unable to determine the directions of change in $\gamma_{3 *}$, $\varphi_{33 *}$, and $\psi_{11 *}$ to $\psi_{33 *}$.

When $x_{2}$ needs to load on $f_{2}$ with $\lambda_{220}>0$, the extra association between $x_{2}$ and $f_{2}$ has to be explained by $m_{x_{2} f_{2}}=\lambda_{2} \gamma_{1} \phi_{11}$; thus $\lambda_{2 *} \gamma_{1 *} \phi_{11 *}$ will be greater. Notice that $m_{x_{1} f_{2}}=\gamma_{1} \phi_{11}$ and $m_{x_{3} f_{2}}=\lambda_{3} \gamma_{1} \phi_{11}, \gamma_{1 *} \phi_{11 *}$ cannot be too much greater, so $\lambda_{2 *}$ has to be much greater. A much greater $\lambda_{2 *}$ needs smaller $\phi_{11 *}$ and $\lambda_{3 *}$ to explain the associations within $\left(x_{1}, x_{2}, x_{3}\right)$. A smaller $\phi_{11 *}$ leads to greater $\gamma_{1 *}, \gamma_{2 *}, \psi_{11 *}$, and $\psi_{33 *}$. We are unable to determine the directions of change in $\gamma_{3 *}, \varphi_{22 *}, \varphi_{33 *}$, and $\psi_{22 *}$.

When $x_{2}$ needs to load on $f_{3}$ with $\lambda_{230}>0$, the extra association between $x_{2}$ and $f_{3}$ has to be explained by $m_{x_{2} f_{3}}$. By similar logic as when $\lambda_{220}>0, \lambda_{2 *}$ has to be much greater; $\phi_{11 *}$ and $\lambda_{3 *}$ have to be smaller; and $\gamma_{1 *}, \gamma_{2 *}, \psi_{11 *}$, and $\psi_{33 *}$ have to be greater. We are unable to determine the directions of change in $\gamma_{3 *}, \varphi_{22 *}, \varphi_{33 *}$, and $\psi_{22 *}$.

Extra factor loadings for $x_{4}$ or $x_{5}$. When $x_{4}$ or $x_{5}$ needs to load on $f_{1}$ or $f_{3}$, the model represented by Figure 6 is also misspecified. The withinand between-cluster covariances of $\left(x_{1}, x_{2}, x_{3}\right)$ and $\left(x_{7}, x_{8}, x_{9}\right)$ are not affected. Thus, $\lambda_{2 *}, \lambda_{3 *}, \lambda_{8 *}, \lambda_{9 *}, \psi_{11 *}$ to $\psi_{33 *}$ and $\psi_{77 *}$ to $\psi_{99 *}$ still equal those corresponding to the correctly specified model, as indicated in Table 5. The variances and covariance $\phi_{11 *}, m_{f_{1} f_{3} *}$, and $m_{f_{3} f_{3} *}$ will also be correctly evaluated.

When $x_{4}$ needs to load on $f_{1}$ with $\lambda_{410}>0$, the stronger association between $x_{4}$ and $f_{1}$ has to be explained by $m_{x_{4} f_{1}}=\gamma_{1} \phi_{11}$, thus $\gamma_{1 *}$ has to be much greater. A greater $\gamma_{1 *}$ needs smaller $\lambda_{5 *}$ and $\lambda_{6 *}$ to explain the intact covariances of $\left(x_{5}, x_{6}\right)$ with $\left(x_{1}, x_{2}, x_{3}\right)$. A much greater $\gamma_{1 *}$ most likely needs to have smaller $\gamma_{2 *}$ and $\gamma_{3 *}$ to keep $m_{f_{1} f_{3}}=\phi_{110}\left(\gamma_{2 *}+\gamma_{1 *} \gamma_{3 *}\right)$ the same. We are unable to determine the directions of change in $\varphi_{22 *}, \varphi_{33 *}$, and $\psi_{44 *}$ to $\psi_{66 *}$.

When $x_{4}$ needs to load on $f_{3}$ with $\lambda_{430}>0$, the extra association between $x_{4}$ and $f_{3}$ has to be explained by $m_{x_{4} f_{3} *}$. Greater $m_{x_{4} f_{3} *}$ leads to smaller $\lambda_{5 *}$ and $\lambda_{6 *}$ to explain the intact covariances of $\left(x_{5}, x_{6}\right)$ with $\left(x_{7}, x_{8}, x_{9}\right)$. Smaller $\lambda_{5 *}$ and $\lambda_{6 *}$ need a greater $\gamma_{1 *}$ to explain the between-cluster associations of $\left(x_{4}, x_{5}, x_{6}\right)$ with $\left(x_{1}, x_{2}, x_{3}\right)$. A greater $\gamma_{1 *}$ needs to have smaller $\gamma_{2 *}$ or $\gamma_{3^{*}}$ or both to keep $m_{f_{1} f_{3} *}$ unchanged. It is difficult for us to determine which case. We are unable to determine the directions of change in $\varphi_{22 *}, \varphi_{33 *}$, and $\psi_{44 *}$ to $\psi_{66 *}$.

When $x_{5}$ needs to load on $f_{1}$ with $\lambda_{510}>0$, the extra association between $x_{5}$ and $f_{1}$ has to be explained by $m_{x_{5} f_{1}}=\lambda_{5} \gamma_{1} \phi_{11}$, thus $\lambda_{5 *} \gamma_{1 *} \phi_{110}$ will be greater. 
Because $f_{1} \rightarrow f_{2}$ is the direct path for the extra association, $\gamma_{1 *}$ will be greater but cannot be too much greater, because $m_{x_{4} f_{1}}=\gamma_{1} \phi_{11}$ and $m_{x_{6} f_{1}}=\lambda_{6} \gamma_{1} \phi_{11}$, so $\lambda_{5 *}$ has to be much greater. A much greater $\lambda_{5 *}$ needs smaller $m_{f_{2} f_{2} *}=$ $\gamma_{1 *}^{2} \phi_{110}+\varphi_{22 *}$ and $\lambda_{6 *}$ to explain the associations within $\left(x_{4}, x_{5}, x_{6}\right)$. Because $\gamma_{1 *}$ is mainly responsible for the between-cluster associations of $\left(x_{1}, x_{2}, x_{3}\right)$ with $\left(x_{4}, x_{5}, x_{6}\right), \varphi_{22 *}$ has to be smaller. A smaller $m_{f_{2} f_{2} *}$ leads to greater $\psi_{44 *}$ and $\psi_{66 *}$. We are unable to determine the directions of change in $\gamma_{2 *}, \gamma_{3 *}, \varphi_{33 *}$, and $\psi_{55 *}$.

When $x_{5}$ needs to load on $f_{3}$ with $\lambda_{530}>0$, the extra association between $x_{5}$ and $f_{3}$ has to be explained by $m_{x_{5} f_{3}}=\lambda_{5} m_{f_{2} f_{3}}$. By an analysis similar to when $\lambda_{510}>0, \lambda_{5 *}$ has to be much greater. A much greater $\lambda_{5 *}$ needs smaller $m_{f_{2} f_{2} *}=\gamma_{1 *}^{2} \phi_{11 *}+\varphi_{22 *}$ and $\lambda_{6 *}$ to explain the associations within $\left(x_{4}, x_{5}, x_{6}\right)$. A smaller $m_{f_{2} f_{2} *}$ needs greater $\gamma_{3 *}, \psi_{44 *}$, and $\psi_{66 *}$. We are unable to determine the directions of change in $\gamma_{1 *}, \gamma_{2 *}, \varphi_{22 *}, \varphi_{33 *}$, and $\psi_{55 *}$.

Extra factor loadings for $x_{7}$ or $x_{8}$. When $x_{7}$ or $x_{8}$ needs to load on $f_{1}$ or $f_{2}$, the model represented by Figure 6 is again misspecified. The within- and between-cluster associations of $\left(x_{1}, x_{2}, x_{3}\right)$ and $\left(x_{4}, x_{5}, x_{6}\right)$ will not be affected. Thus, $\lambda_{2 *}, \lambda_{3 *}, \lambda_{5 *}, \lambda_{6 *}$, and $\psi_{11 *}$ to $\psi_{66 *}$ still equal the population values corresponding to the correctly specified model, as indicated in Table 5. The variances and covariance $m_{f_{1} f_{1} *}, m_{f_{1} f_{2} *}$, and $m_{f_{2} f_{2} *}$ will also be correctly evaluated with $\phi_{11 *}=\phi_{110}, \gamma_{1 *}=\gamma_{10}$, and $\varphi_{22 *}=\varphi_{220}$.

When $x_{7}$ needs to load on $f_{1}$ with $\lambda_{710}>0$, the extra association has to be explained by $m_{x_{7} f_{1}}=m_{f_{3} f_{1}}=\phi_{11}\left(\gamma_{2}+\gamma_{1} \gamma_{3}\right)$. Being mainly responsible for the association between $f_{1}$ and $f_{3}, \gamma_{2 *}$ has to be greater. Smaller $\lambda_{8 *}$ and $\lambda_{9 *}$ are needed to explain the associations of $\left(x_{8}, x_{9}\right)$ with $\left(x_{1}, x_{2}, x_{3}\right)$. We are unable to determine the directions of change in $\gamma_{3 *}, \varphi_{33 *}$, and $\psi_{77 *}$ to $\psi_{99 *}$.

When $x_{7}$ needs to load on $f_{2}$ with $\lambda_{720}>0$, the extra association has to be explained by $m_{x_{7} f_{2}}=m_{f_{3} f_{2}}$. Being mainly responsible for the path $f_{2} \rightarrow f_{3}$, $\gamma_{3 *}$ should be greater. A greater $m_{f_{3} f_{2} *}$ needs smaller $\lambda_{8 *}$ and $\lambda_{9 *}$ to properly explain the between-cluster associations of $\left(x_{7}, x_{8}, x_{9}\right)$ with $\left(x_{4}, x_{5}, x_{6}\right)$. Smaller $\lambda_{8 *}$ and $\lambda_{9 *}$ need greater $\gamma_{2 *}$ for the between-cluster associations of $\left(x_{1}, x_{2}, x_{3}\right)$ and $\left(x_{7}, x_{8}, x_{9}\right)$. We are unable to determine the directions of change in $\varphi_{33 *}$ and $\psi_{77 *}$ to $\psi_{99 *}$.

When $x_{8}$ needs to load on $f_{1}$ with $\lambda_{810}>0$, the extra association has to be explained by $m_{x_{8} f_{1}}=\lambda_{8} m_{f_{3} f_{1}}$. Being mainly responsible for the association between $f_{1}$ and $f_{3}, \gamma_{2 *}$ has to be greater. Because $m_{f_{3} f_{1}}$ is shared by all the variables in $\left(x_{1}, x_{2}, x_{3}\right)$ and $\left(x_{7}, x_{8}, x_{9}\right)$ for between-cluster covariances, $\lambda_{8 *}$ has to be much greater. A much greater $\lambda_{8 *}$ needs smaller $\lambda_{9 *}$ and $m_{f_{3} f_{3} *}$ to explain the within-cluster associations of $\left(x_{1}, x_{2}, x_{3}\right)$, which further lead to greater $\psi_{77 *}$ and $\psi_{99 *}$. We are unable to determine the directions of change in $\gamma_{3 *}, \varphi_{33 *}$, and $\psi_{88 *}$. 
When $x_{8}$ needs to load on $f_{2}$ with $\lambda_{820}>0$, the extra association has to be explained by $m_{x_{8} f_{2}}=\lambda_{8} m_{f_{3} f_{2}}$. Being mainly responsible for the association between $f_{3}$ and $f_{2}, \gamma_{3 *}$ has to be greater. Because $m_{f_{3} f_{2}}$ is shared by $\left(x_{4}, x_{5}, x_{6}\right)$ and $\left(x_{7}, x_{8}, x_{9}\right)$ for their between-cluster associations, $\lambda_{8 *}$ has to be much greater. A greater $\lambda_{8 *}$ needs smaller $\lambda_{9 *}$ and $m_{f_{3} f_{3} *}$ to explain the within-cluster associations of $\left(x_{7}, x_{8}, x_{9}\right)$, which further leads to greater $\psi_{77 *}$ and $\psi_{99 *}$. A much greater $\lambda_{8 *}$ most likely needs a smaller $\gamma_{2 *}$ for the between-cluster associations of $\left(x_{1}, x_{2}, x_{3}\right)$ and $\left(x_{7}, x_{8}, x_{9}\right)$. We are unable to determine the directions of change in $\varphi_{33 *}$ and $\psi_{88 *}$.

Excluding the $\gamma_{j}$. The remaining analysis for Figure 6 is when one of the paths among the latent factors $f_{1}, f_{2}$, and $f_{3}$ are excluded. Similar to the previous analyses, we consider cases when a positive $\gamma_{j}$ exists in the population but not in the model. We discuss the effect in the order of excluding $\gamma_{1}, \gamma_{2}$, and $\gamma_{3}$. The results of the analysis are summarized in Table 6 , where $\times$ means the parameter is fixed at zero or the path represented by that parameter is excluded from the model.

TABLE 6

The Directions of Change in Parameters

When $\gamma_{j}>0$ Exists in the Population

But Is Excluded From the Model in Figure 6

\begin{tabular}{llll}
\hline$\theta_{*}$ & $\gamma_{1}$ & $\gamma_{2}$ & $\gamma_{3}$ \\
\hline$\lambda_{2 *}$ & 0 & 0 & $?$ \\
$\lambda_{3 *}$ & 0 & 0 & $?$ \\
$\lambda_{5 *}$ & 0 & $?$ & 0 \\
$\lambda_{6 *}$ & 0 & $?$ & 0 \\
$\lambda_{8 *}$ & 0 & 0 & 0 \\
$\lambda_{9 *}$ & 0 & 0 & 0 \\
$\phi_{11 *}$ & 0 & 0 & $?$ \\
$\gamma_{1 *}$ & $\times$ & + & + \\
$\gamma_{2 *}$ & + & $\times$ & + \\
$\gamma_{3 *}$ & + & + & $\times$ \\
$\varphi_{22 *}$ & + & - & - \\
$\varphi_{33 *}$ & $?$ & $?$ & $?$ \\
$\psi_{11 *}$ & 0 & 0 & $?$ \\
$\psi_{22 *}$ & 0 & 0 & $?$ \\
$\psi_{33 *}$ & 0 & 0 & $?$ \\
$\psi_{44 *}$ & 0 & $?$ & 0 \\
$\psi_{55 *}$ & 0 & $?$ & 0 \\
$\psi_{66 *}$ & 0 & $?$ & 0 \\
$\psi_{77 *}$ & 0 & 0 & 0 \\
$\psi_{88 *}$ & 0 & 0 & 0 \\
$\psi_{99 *}$ & 0 & 0 & 0 \\
\hline
\end{tabular}


When $\gamma_{10}>0$ in Figure 6 and the path $f_{1} \rightarrow f_{2}$ is removed from the model, let $m_{f_{2} f_{2}}=\phi_{22}=\varphi_{22}$. The model-implied covariance matrix of $\left(f_{1}, f_{2}, f_{3}\right)$ can be obtained from the matrix in Equation 2 by letting $\gamma_{1}=0$. It follows from either the model-implied covariance matrix or the path diagram that the values of $\phi_{11}$ and $\gamma_{2}$ are not identified in $m_{f_{1} f_{3}}=\phi_{11} \gamma_{2}$. Similarly, the values of $\phi_{22}$ and $\gamma_{3}$ are confounded in $m_{f_{2} f_{3}}=\phi_{22} \gamma_{3}$. Because $m_{f_{1} f_{2}}=0$ whatever values the parameters are, $\phi_{11 *}$ will adjust for the covariances within $\left(x_{1}, x_{2}, x_{3}\right)$ and $\gamma_{2 *}$ will take whatever $\phi_{11 *}$ is and adjust for the covariance between $\left(x_{1}, x_{2}, x_{3}\right)$ and $\left(x_{7}, x_{8}, x_{9}\right)$. Thus, $\phi_{11 *}=\phi_{110}, \lambda_{2 *}=\lambda_{20}$, and $\lambda_{3 *}=\lambda_{30}$. Similarly, $\lambda_{5 *}=\lambda_{50}$, $\lambda_{6 *}=\lambda_{60}$, and $\varphi_{22 *}=\phi_{22 *}=\sigma_{f_{2} f_{2}}=\gamma_{10}^{2} \phi_{110}+\varphi_{220}$. The only function of $\varphi_{33}$ is to adjust for the variance of $f_{3}$, as being predicted by $f_{1}$ and $f_{2}$, the between-cluster covariances can and have to be explained by $\gamma_{2 *}$ and $\gamma_{3 *}$, so $\lambda_{8 *}=\lambda_{80}$ and $\lambda_{9 *}=\lambda_{90}$. The parameters $\psi_{i i *} \mathrm{~s}$ also equal their counterparts corresponding to the correctly specified model. Notice that in the population there exists $\sigma_{f_{1} f_{3}}>\phi_{110} \gamma_{20}$, hence $\gamma_{2 *}$ has to be greater to properly explain the covariances between $\left(x_{1}, x_{2}, x_{3}\right)$ and $\left(x_{7}, x_{8}, x_{9}\right)$. Similarly, $\sigma_{f_{2} f_{3}}>\phi_{22 *} \gamma_{30}, \gamma_{3 *}$ has to be greater to explain the covariances between $\left(x_{4}, x_{5}, x_{6}\right)$ and $\left(x_{7}, x_{8}, x_{9}\right)$. We are unable to predict the direction of change in $\varphi_{33 *}$.

When $\gamma_{20}>0$ in Figure 6 and the path $f_{1} \rightarrow f_{3}$ is removed from the model, the model-implied covariance matrix of $\left(f_{1}, f_{2}, f_{3}\right)$ can be obtained from the matrix in Equation 2 by letting $\gamma_{2}=0$. Similar to when $f_{1} \rightarrow f_{2}$ is removed, $\gamma_{1}$ and $\phi_{11}$ are confounded in explaining the relation among the factors, $\phi_{11 *}$ will equal $\phi_{110}$ to explain the covariances within $\left(x_{1}, x_{2}, x_{3}\right)$, and $\gamma_{1 *}$ will take a value to best represent the between-cluster associations. Similarly, $\varphi_{33} *$ will adjust for $m_{f_{3} f_{3} *}$ to fully explain the covariance within the cluster $\left(x_{7}, x_{8}, x_{9}\right)$. Thus, $\lambda_{2 *}$, $\lambda_{3 *}, \lambda_{8 *}, \lambda_{9 *}, \psi_{11 *}$ to $\psi_{33 *}$, and $\psi_{77 *}$ to $\psi_{99 *}$ will equal their counterparts corresponding to the correctly specified model. When $f_{1} \rightarrow f_{3}$ existed, the association between $f_{1}$ and $f_{3}$ was mostly through the direct path; now, it has to be through the indirect path. Although the path $f_{2} \rightarrow f_{3}$ still exists, due to missing $f_{1} \rightarrow f_{3}$, now $m_{f_{2} f_{3}}=\gamma_{1}^{2} \gamma_{3} \phi_{11}+\gamma_{3} \varphi_{22}$. It follows from the matrix in Equation 2 that, in the population, $\sigma_{f_{2} f_{3}}>\gamma_{10}^{2} \gamma_{30} \phi_{110}+\gamma_{30} \varphi_{220}$. With $\phi_{11 *}=\phi_{110}$, both $\gamma_{1 *}$ and $\gamma_{3 *}$ have to be greater to balance the effect of the excluded path. Notice that the main and direct effect of $\varphi_{22}$ is to adjust for $m_{f_{2} f_{2}}$ although it also contributes to $m_{f_{2} f_{3}}$. Due to a greater $\gamma_{1 *}, \varphi_{22 *}$ should become smaller. We are unable to determine the directions of change in $\lambda_{5 *}, \lambda_{6 *}, \psi_{44 *}$ to $\psi_{66 *}$, and $\varphi_{33 *}$.

When $\gamma_{30}>0$ in Figure 6 and the path $f_{2} \rightarrow f_{3}$ is removed from the model, the model-implied covariance matrix of $\left(f_{1}, f_{2}, f_{3}\right)$ can be obtained from Equation 2 by letting $\gamma_{3}=0$. Now, the only function of $\varphi_{22}$ is to adjust for $m_{f_{2} f_{2}}$, so the parameters within the cluster $\left(x_{4}, x_{5}, x_{6}\right)$ can still be evaluated correctly. For the same reason, the parameters within the cluster $\left(x_{7}, x_{8}, x_{9}\right)$ will be evaluated correctly. Without the path $f_{2} \rightarrow f_{3}$, the covariance between $f_{2}$ and 
$f_{3}$ is explained by their sharing the same predictor $f_{1}$, with the model-implied covariance $m_{f_{2} f_{3}}=\phi_{11} \gamma_{1} \gamma_{2}$. In Figure 6 , there are two paths for $f_{1}$ and $f_{3}$ to correlate, one is the direct path $f_{2} \rightarrow f_{3}$, and the other is the indirect path $f_{1} \rightarrow f_{2} \rightarrow f_{3}$. Without $f_{2} \rightarrow f_{3}$, the direct path will pick up the association in the indirect path. Actually, in the covariance matrix in Equation 2, $\gamma_{2}$ always appears in the form $\gamma_{2}+\gamma_{1} \gamma_{3}$. Thus, without $\gamma_{3}, \gamma_{2 *}$ will at least increase up to $\gamma_{20}+\gamma_{10} \gamma_{30}$. However, an increased $\gamma_{2 *}$ alone is not enough for modeling $\sigma_{f_{2} f_{3}}=\gamma_{10} \phi_{110}\left(\gamma_{20}+\gamma_{10} \gamma_{30}\right)+\gamma_{30} \varphi_{220}$. Notice that $\gamma_{1}$ and $\gamma_{2}$ are parallel in modeling $\sigma_{f_{2} f_{3}}$. Because $\phi_{11 *}$ needs to model the covariances within $\left(x_{1}, x_{2}, x_{3}\right)$, both $\gamma_{1 *}$ and $\gamma_{2 *}$ need to be greater. Due to the parallel positions of $\gamma_{1}$ and $\gamma_{2}$, a greater $m_{f_{2} f_{3} *}=\gamma_{1 *} \gamma_{2 *} \phi_{11 *}$ most likely leads to a greater $\gamma_{1 *}^{2} \phi_{11 *}$, and thus a smaller $\varphi_{22 *}$. A greater $\gamma_{1 *}$ may need a smaller $\phi_{11 *}$ or $\lambda_{2 *}$ or $\lambda_{3 *}$ to explain the covariances between $\left(x_{1}, x_{2}, x_{3}\right)$ and $\left(x_{4}, x_{5}, x_{6}\right)$. We are unable to determine the directions of change in $\phi_{11 *}, \lambda_{2 *}, \lambda_{3 *}, \varphi_{33 *}$, and $\psi_{11 *}$ to $\psi_{33 *}$.

\section{EXAMPLES}

The previous sections illustrated the technique of analyzing the path to identify parameter changes due to model misspecification. This section applies the technique to three models. The first one is a continuation of Example 1, in which we show that the predicted change in $\gamma_{3 *}$ by analyzing the path agrees with the change in $\hat{\gamma}_{3}$ when the model is changed. The second one is based on a data set from Chatterjee, Handcock, and Simonoff (1995); the model is a one-factor model. We verify whether the parameter change as predicted by analyzing the path concurs with that in real data. The last one is based on a data set from Holzinger and Swineford (1939). That model is a confirmatory threefactor model, the same as the one analyzed earlier. Because we do not know the correct model in any of the examples, our analysis is to compare parameter estimates between different models. These examples allow us to see how the technique of analyzing the path performs with real data, especially whether it can still predict the change of parameter estimates when the model with more parameters may only represent a closer fit.

\section{Example 1 Continued}

Applying the same analysis as for the three-factor structural equation model in the previous section to Figure $1, \gamma_{3 *}$ should increase when (a) $e_{3} \leftrightarrow e_{5}$ is removed; (b) $e_{4} \leftrightarrow e_{6}$ is removed; (c) both $e_{3} \leftrightarrow e_{5}$ and $e_{4} \leftrightarrow e_{6}$ are removed; and (d) $e_{3} \leftrightarrow e_{5}$ and $e_{4} \leftrightarrow e_{6}$ are removed, as well as when $f_{1}$ together with education and occupational status index are out of the model. With the real data, 
the maximum likelihood estimates $\hat{\gamma}_{3} \mathrm{~s}$ are

\begin{tabular}{c|ccccc}
\hline model change & (a) & (b) & (c) & (d) & (e) \\
\hline$\hat{\gamma}_{3}$ & 0.655 & 0.617 & 0.705 & 0.789 & 0.607 \\
$p$ & 0.000 & 0.275 & 0.000 & 0.000 & 0.316 \\
\hline
\end{tabular}

where (e) is for the full model in Figure 1. Our analysis therefore applies not only to the population parameter values, but also to their estimates. The second row of numbers are the $p$ values when referring the normal distribution-based likelihood ratio statistics, with $N=932$, to the corresponding chi-square distributions. Both the full model and the model without the path $e_{4} \leftrightarrow e_{6}$ are statistically acceptable. However, they generate different $\hat{\gamma}_{3}$. If one is an unbiased estimate, the other might be a biased estimate. A complication with real data is that the difference between the different $\hat{\gamma}_{3}$ s might be just due to sampling error. Yuan et al. (2003) provided a procedure for testing the significance of the difference.

\section{Example 2}

Chatterjee et al. (1995, p. 299) contains a data set of descriptive statistics for 105 guards of the National Basketball Association (NBA) for the 1992-1993 season. Among the variables are total minutes played, points scored per game, assists per game, and rebounds per game. Yuan and Bentler (1998) proposed a one-factor model for these four variables. To make the four variables have comparable standard deviations we divide the first three variables by 880,6 , and 2 , respectively. Such a change of scale makes the convergence of the iterative procedure for obtaining the maximum likelihood estimates much faster. It has no effect on the substantive aspects of the analysis but makes the factor loadings and error variances of similar magnitude.

Fitting the sample covariance matrix to a one-factor model, as represented in Figure 2 (without the correlated error), leads to the first column of numbers in Table 7. However, the LM test in EQS indicates that the model will fit the data significantly better if allowing $e_{2}$ and $e_{4}$ to correlate. Adding the parameter $\psi_{24}$ to the model results in the second column of numbers under $\hat{\boldsymbol{\theta}}_{0}$ in Table 7. Due to the model change, the likelihood ratio statistic changes from 15.281 with $2 d f$ to 3.557 with $1 d f$; the LM test implies that no other model modification is needed at the .05 level. If we regard the modified model as the correct model, then $\hat{\boldsymbol{\theta}}-\hat{\boldsymbol{\theta}}_{0}$ contains both the sampling error and the biases. The fourth column, under $D$, is the signs of bias obtained by analyzing the path, which agree with the actual change on all the estimates.

Yuan and Bentler (1998) noted that cases numbered 2, 4, and 6 in the NBA data are influential and should be downweighted or removed for proper analysis. After the three cases are removed, the parallel results are shown on the right 
TABLE 7

Maximum Likelihood Estimates for Two Different Confirmatory Factor Models Using NBA Data and by Removing Three Cases

\begin{tabular}{|c|c|c|c|c|c|c|c|c|}
\hline \multirow[b]{2}{*}{$\theta$} & \multicolumn{4}{|c|}{ The Original Sample } & \multicolumn{4}{|c|}{ Cases 2, 4, 6 Removed } \\
\hline & $\hat{\boldsymbol{\theta}}$ & $\hat{\boldsymbol{\theta}}_{0}$ & $\hat{\boldsymbol{\theta}}-\hat{\boldsymbol{\theta}}_{0}$ & $D$ & $\hat{\boldsymbol{\theta}}$ & $\hat{\boldsymbol{\theta}}_{0}$ & $\hat{\boldsymbol{\theta}}-\hat{\boldsymbol{\theta}}_{0}$ & $D$ \\
\hline$\lambda_{1}$ & 0.897 & 0.977 & -0.080 & - & 0.911 & 0.959 & -0.048 & - \\
\hline$\lambda_{2}$ & 0.895 & 0.818 & 0.078 & + & 0.805 & 0.759 & 0.046 & + \\
\hline$\lambda_{3}$ & 0.895 & 0.913 & -0.018 & - & 0.937 & 0.945 & -0.008 & - \\
\hline$\lambda_{4}$ & 1.086 & 0.959 & 0.127 & + & 1.004 & 0.937 & 0.067 & + \\
\hline$\psi_{11}$ & 0.208 & 0.058 & 0.150 & + & 0.160 & 0.070 & 0.089 & + \\
\hline$\psi_{22}$ & 0.218 & 0.351 & -0.133 & - & 0.197 & 0.268 & -0.071 & - \\
\hline$\psi_{33}$ & 0.749 & 0.716 & 0.033 & + & 0.704 & 0.690 & 0.014 & + \\
\hline$\psi_{44}$ & 0.478 & 0.737 & -0.260 & - & 0.355 & 0.485 & -0.130 & - \\
\hline$\psi_{24}$ & $x$ & 0.238 & & & $\times$ & 0.131 & & \\
\hline
\end{tabular}

side of Table 7. Again, the predicted change and the actual change in parameter estimates agree. Without Cases 2, 4, and 6, the LM test also implies that adding the parameter $\psi_{24}$ can improve the model fit significantly; the likelihood ratio statistic changes from 7.500 to 1.201 due to the model change.

\section{Example 3}

Holzinger and Swineford (1939) contains test scores on the following subtests or variables: visual perception, cubes, lozenges, paragraph comprehension, sentence completion, word meaning, addition, counting dots, and straight-curved capitals. The first three variables were designed to measure spatial ability, the next three variables were designed to measure verbal ability, and the last three variables were administered with a limited time and were designed to measure a speed factor in performing the tasks. Thus, Holzinger and Swineford's design can be represented by the confirmatory factor model in Figure 4. However, all nine available statistics for testing $\boldsymbol{\Sigma}=\mathbf{M}(\boldsymbol{\theta})$ indicate that the model does not fit the sample (see Yuan \& Bentler, 2007a), the largest $p$ value is .015. The LM test indicates that the model would fit the data much better when adding either $e_{7} \leftrightarrow e_{8}$ or $x_{9} \leftarrow f_{1}$ to the model represented by Figure 4 . Assuming the model with a free $\psi_{78}$ is correct, parameter estimates $\hat{\lambda}_{7}, \hat{\lambda}_{8}$, and $\hat{\psi}_{99}$ will contain positive biases according to our analysis; $\hat{\lambda}_{9}, \hat{\phi}_{13}, \hat{\phi}_{23}, \hat{\psi}_{77}$, and $\hat{\psi}_{88}$ will contain negative biases; the rest of the parameter estimates do not contain biases. Assuming the model with a free $\lambda_{91}$ is correct, then $\hat{\lambda}_{9}, \hat{\phi}_{13}, \hat{\psi}_{77}$, and $\hat{\psi}_{88}$ will contain positive biases; and $\hat{\lambda}_{7}$ and $\hat{\lambda}_{8}$ will contain negative biases; and $\hat{\lambda}_{1}$ 
to $\hat{\lambda}_{6}$ and $\hat{\psi}_{11}$ to $\hat{\psi}_{66}$ do not contain any biases. These results will be contrasted with numerical values of the estimates next.

Because standard deviations of the nine variables differ a lot, we divide them, respectively, by $6,4,8,3,4,7,23,20,36$ to keep each marginal standard deviation between 1 and 2 . This change of scale makes the factor loadings and error variances of a similar magnitude; thus, it facilitates our comparison of parameter change. Table 8 contains the maximum likelihood estimates $\hat{\boldsymbol{\theta}}$, corresponding to the model represented in Figure 4; and $\hat{\boldsymbol{\theta}}_{01}$, corresponding to the model with $e_{7} \leftrightarrow e_{8}$ being added to Figure 4 and $\hat{\boldsymbol{\theta}}_{02}$, corresponding to the model with $x_{9} \leftarrow f_{1}$ being added to Figure 4. For easy comparison, the differences $\hat{\boldsymbol{\theta}}-\hat{\boldsymbol{\theta}}_{01}$ and $\hat{\boldsymbol{\theta}}-\hat{\boldsymbol{\theta}}_{02}$ as well as the directions of change in $\theta_{*}$, under $D$, are also included in Table 8 . For all the $\theta_{*}$ s that are going to change according to the analysis, their estimates move in the predicted direction. For those $\theta_{*}=\theta_{0}$ according to the analysis, their estimates also become smaller or larger, most likely due to sampling error. For those the analysis cannot predict, their change might be due to sampling error as well as biases.

TABLE 8

Maximum Likelihood Estimates for Three Different Confirmatory Factor Models Using Data of Holzinger and Swineford (1939)

\begin{tabular}{lcccrrrr}
\hline$\theta$ & $\hat{\boldsymbol{\theta}}$ & $\hat{\boldsymbol{\theta}}_{01}$ & $\hat{\boldsymbol{\theta}}_{02}$ & $\hat{\boldsymbol{\theta}}-\hat{\boldsymbol{\theta}}_{01}$ & $D$ & $\hat{\boldsymbol{\theta}}-\hat{\boldsymbol{\theta}}_{02}$ & $D$ \\
\hline$\lambda_{1}$ & 0.779 & 0.821 & 0.819 & -0.041 & 0 & -0.040 & 0 \\
$\lambda_{2}$ & 0.574 & 0.548 & 0.543 & 0.026 & 0 & 0.031 & 0 \\
$\lambda_{3}$ & 0.721 & 0.693 & 0.688 & 0.028 & 0 & 0.033 & 0 \\
$\lambda_{4}$ & 0.974 & 0.975 & 0.975 & -0.001 & 0 & -0.001 & 0 \\
$\lambda_{5}$ & 0.964 & 0.965 & 0.964 & -0.001 & 0 & 0.000 & 0 \\
$\lambda_{6}$ & 0.938 & 0.936 & 0.937 & 0.002 & 0 & 0.001 & 0 \\
$\lambda_{7}$ & 0.682 & 0.440 & 0.708 & 0.242 & + & -0.026 & - \\
$\lambda_{8}$ & 0.837 & 0.568 & 0.900 & 0.269 & + & -0.063 & - \\
$\lambda_{9}$ & 0.720 & 1.007 & 0.453 & -0.287 & - & 0.267 & + \\
$\phi_{12}$ & 0.541 & 0.542 & 0.554 & -0.001 & 0 & -0.013 & 0 \\
$\phi_{13}$ & 0.522 & 0.624 & 0.392 & -0.102 & - & 0.130 & + \\
$\phi_{23}$ & 0.335 & 0.381 & 0.240 & -0.046 & - & 0.096 & $?$ \\
$\psi_{11}$ & 0.721 & 0.654 & 0.657 & 0.066 & 0 & 0.064 & 0 \\
$\psi_{22}$ & 0.905 & 0.934 & 0.940 & -0.029 & 0 & -0.035 & 0 \\
$\psi_{33}$ & 0.560 & 0.601 & 0.607 & -0.040 & 0 & -0.046 & 0 \\
$\psi_{44}$ & 0.317 & 0.315 & 0.315 & 0.002 & 0 & 0.002 & 0 \\
$\psi_{55}$ & 0.422 & 0.421 & 0.422 & 0.001 & 0 & -0.000 & 0 \\
$\psi_{66}$ & 0.409 & 0.413 & 0.411 & -0.004 & 0 & -0.002 & 0 \\
$\psi_{77}$ & 0.604 & 0.876 & 0.568 & -0.272 & - & 0.036 & + \\
$\psi_{88}$ & 0.402 & 0.780 & 0.293 & -0.378 & - & 0.109 & + \\
$\psi_{99}$ & 0.540 & 0.045 & 0.479 & 0.495 & + & 0.062 & $?$ \\
$\psi_{78}$ & $\times$ & 0.388 & $\times$ & & & & \\
$\lambda_{91}$ & $\times$ & $\times$ & 0.460 & & & & \\
\hline & & & & & & & \\
\hline
\end{tabular}


We might have observed that, in Table 8, a parameter or its estimate may change to different directions when the model is modified differently. If the model with $\lambda_{91}$ being included represents the correct model, then $\hat{\lambda}_{7}$ and $\hat{\lambda}_{8}$ contain negative biases, whereas $\hat{\lambda}_{9}, \hat{\phi}_{13}, \hat{\psi}_{77}$, and $\hat{\psi}_{88}$ contain positive biases. If the model with $\psi_{78}$ being included represents the correct model, then these estimates contain biases in the opposite directions. This is because the two modified models are not mathematically equivalent. Actually, all the reliable statistics indicate both the modified models fit the data well. Again, the challenge with practical data is that we do not know which model is the correct one.

\section{DISCUSSION}

SEM, and covariance structural analysis in particular, has been widely used in the social and behavior sciences. Due to the effort of many methodologists, the capacity of SEM in modeling more complex data structures is increasing. When simultaneously modeling the relation among manifest and latent variables as well as measurement errors, misspecification is inevitable. Diagnostic tools that facilitate the understanding of the relation among different parts of the model and how misspecification affects model parameters are needed. Advanced mathematical and statistical procedures have been developed to study the effect of misspecification (e.g., Yuan et al., 2003), but they are not designed for model diagnosis. The technique of analyzing the path is not only intuitive but also facilitates a good understanding of the relation of various parts of the model and provides a clear picture of reactions of parameters to a misspecification. Thus, it is a valuable tool for model diagnosis. However, like any other method, analyzing the path needs conditions to work well. We discuss these conditions and related results later.

We intend to introduce the technique of analyzing the path for model diagnosis. The mathematics behind the analysis is Equation 1, which holds only when $\boldsymbol{\Sigma}$ is in a neighborhood of $\boldsymbol{\Sigma}_{0}$. The validity of analyzing the path is also restricted to when $\Sigma$ is close to $\Sigma_{0}$. Notice that the coefficients $a_{i j}$ s in Equation 1 depend on the discrepancy function used in estimating the model, so analyzing the path also depends on the discrepancy function employed. Actually, the function $\mathbf{g}(\boldsymbol{\Sigma})$ introduced earlier is closely related to the weight matrix in the estimating equation (Yuan et al., 2003). Among all the discrepancy functions, only the normal distribution-based likelihood function corresponds to a weight matrix decided solely by the structural model $\mathbf{M}(\boldsymbol{\theta})$. When the model is misspecified, different discrepancy functions are not equivalent (Yuan \& Chan, 2005). Because analyzing the path only uses information from the model and not from the weight matrix, the results obtained only correspond to the most widely used ML method. Of course, the function $\boldsymbol{\theta}=\mathbf{g}(\Sigma)$ also depends on $\mathbf{M}(\boldsymbol{\theta})$. Thus, 
the $a_{i j}$ in Equation 1 is model dependent, which implies that analyzing the path is also model specific. There is no general conclusion that a parameter has to become smaller or greater when either $\boldsymbol{\Sigma}$ or $\mathbf{M}$ changes arbitrarily. When $\mathbf{M}$ is given, $\Delta \theta=\Delta \theta(\boldsymbol{\Sigma})$ is just a function of $\boldsymbol{\Sigma}$. When $\sigma_{i j}$ is perturbed, the bias $\Delta \theta$ is characterized by Equation 1 and is predictable by analyzing the path. The models discussed in this article aim to be simple and to mimic those typically encountered in textbooks. The same technique and logic can be equally applied to more complex situations. For example, with more indicators in a cluster, the corresponding $\theta_{*}$ will also change according to the analysis but the actual values of the change may be smaller due to their subjection to more model and population constraints. Equality constrains are often used in SEM. When parameters $\theta_{1}$ and $\theta_{2}$ are set equal but their population values $\theta_{10}$ and $\theta_{20}$ in the unrestricted model are not equal, then their common estimated value in the restricted model will be somewhere between $\theta_{10}$ and $\theta_{20}$.

We have only discussed biases in unstandardized parameter estimates due to model misspecification. In the applied literature, standardized estimates are reported more often than unstandardized estimates. Because standardized estimates are functions of unstandardized estimates, biases in unstandardized parameter estimates imply biases in standardized estimates. Let $\hat{\lambda}_{s i}$ be the standardized estimate of the factor loading $\lambda_{i}$ and $\hat{\psi}_{s i}$ be the standardized estimate of the standard deviation of $e_{i}$ in the model represented by either Figure 2 or 4 . Then

$$
\hat{\lambda}_{s i}=\hat{\lambda}_{i} / s_{i}, \quad \hat{\psi}_{s i}=\hat{\psi}_{i} / s_{i}
$$

where $\hat{\psi}_{i}=\hat{\psi}_{i i}^{1 / 2}$ and $s_{i}$ is the sample standard deviation of $x_{i}$. Because $s_{i}$ is consistent for $\sigma_{i}, \hat{\lambda}_{s i}$ and $\hat{\psi}_{s i}$ are consistent estimates of $\lambda_{s i *}=\lambda_{i *} / \sigma_{i}$ and $\psi_{s i *}=\psi_{i *} / \sigma_{i}$, respectively. Thus, the biases in $\hat{\lambda}_{s i}$ and $\hat{\psi}_{s i}$ will be in the same directions as those in $\hat{\lambda}_{i}$ and $\hat{\psi}_{i i}$, respectively. The simple relation in Equation 3 is due to $\phi_{k k}=1$ in Figures 2 and 4 . When $\phi_{k k} \neq 1$ as in Figures 1, 3, 5, and 6 ,

$$
\hat{\lambda}_{s i}=\hat{\lambda}_{i} \hat{\phi}_{k} / s_{i}, \quad \hat{\psi}_{s i}=\hat{\psi}_{i} / s_{i}
$$

where $\hat{\phi}_{k}=\hat{\phi}_{k k}^{1 / 2}$ is the estimate of the standard deviation of the $k$ th factor. Then the direction of the bias in $\hat{\lambda}_{s i}$ depends on the magnitude of the biases in $\hat{\lambda}_{i}$ and $\hat{\phi}_{k k}$. When $\hat{\lambda}_{i}$ contains a positive bias and $\hat{\phi}_{k k}$ contains a negative bias, the bias in $\hat{\lambda}_{s i}$ can be either negative or positive. So the directions of biases in standardized estimates are more difficult to determine by analyzing the path.

Analyzing the path is not a mathematical proof; the directions of change in parameters or their estimates will most likely happen according to the analysis. 
They may not always happen as predicted under all conditions. First, our analysis is based on a model containing only a single misspecification. When a model contains multiple misspecifications or misses multiple parameters, a parameter may need to become larger by one misspecification and to become smaller by another. The resulting value of the parameter will be the accumulated changes, depending on the specific value of each change. Then analyzing the path cannot predict the direction of the accumulated parameter change. If dealing with one misspecification at a time, even when neither of the models is correct, the result of analyzing the path will still be valuable, as illustrated by the examples in the previous section, where we do not have the true model. Second, outliers may create problems when applying the technique to data. One outlier in a single sample may move a parameter estimate to an arbitrary place, and analyzing the path cannot predict such kinds of biases. When data contain outliers or heavy tails, one may need to remove the outliers, use a robust procedure, or transform the data before applying the ML procedure (see Yuan, Chan, \& Bentler, 2000). Third, our analysis is based on all the model parameter values being comparable. When one parameter has a dominated value, a change in this parameter may have a dominant effect that is different from our analysis. In practice, one may rescale the variables to have comparable standard deviations, as was done to the NBA data and data of Holzinger and Swineford (1939) in the previous section. Fourth, analyzing the path cannot identify the directions of change on all model parameters. This typically happens when $\theta$ is associated with several other parameters in explaining a variance or covariance, some of which need $\theta_{*}$ $(\hat{\theta})$ to be greater and some of which need $\theta_{*}(\hat{\theta})$ to be smaller. Fifth, analyzing the path cannot identify the directions of change on parameters when latent variables are omitted in the model. A structure equation or confirmatory factor analysis model is at least partially justified by theory or previous exploratory analysis, so missing latent variables in the model are possible but unlikely. Most of these limitations are not unique to analyzing the path. For example, a single outlier can move the commonly used likelihood ratio statistic to an arbitrary value (Yuan \& Bentler, 2001). Similarly, a statistical test may not be able to tell whether the population value of a parameter is changed either due to effect size, sampling errors, or crude estimation of standard errors, especially with misspecified models (see Yuan \& Hayashi, 2006). Actually, none of the differences of the estimates in Table 8 is zero. Analyzing the path tells us that some of these nonzero numbers are just due to sampling errors.

Analyzing the path is parallel to the functional relation in Equation 1 but provides an intuitive tool for model diagnosis. Different from the functional relation is the statistical relation that characterizes the reaction (fluctuation) of parameter estimates with sampling errors occurring to all the variables. The fluctuation and correlation among parameter estimates, caused by sampling errors, can be described by the asymptotic covariance matrix of the estimates. 
For normally distributed data, the asymptotic covariance matrix is obtained by inverting the related information matrix. With a particular change in the model, the biases in parameter estimates have little to do with their correlations. For example, with positive population factor loadings in Figure 2 and normally distributed data, their estimates are positively correlated. When a pair of errors in Figure 2 are correlated and ignored, two loading estimates contain positive biases and two contain negative biases. Actually, asymptotic or population correlations or covariances among parameter estimates depend on the distribution of the data. For given $\sigma$ s, the correlations among $\hat{\theta}$ s change when the distribution of the sample changes; the values of $\theta_{*}$ and $\theta_{0}$ remain fixed when an estimation procedure is chosen, regardless of how the population distribution changes. Similarly, the predicted value change of a parameter estimate by analyzing the path does not imply the corresponding standard error will change proportionally.

The focus of this article is the biases in parameter estimates due to model misspecification. For certain parameters in statistical modeling, estimates with small biases may have much smaller variances when compared with those of the unbiased estimates. In the context of ML estimation for covariance structure models, adding variables or reducing the number of parameters usually leads to smaller variances (Kano, Bentler, \& Mooijaart, 1993; Yung \& Bentler, 1999). Biased estimates due to model misspecification do not necessarily have smaller variances (Yuan \& Hayashi, 2006).

In summary, analyzing the path is an intuitive tool for model diagnosis for the most widely used ML procedure. It not only provides information on biases of parameter estimates when a model is misspecified but also facilitates a good understanding of the relation of various parts of the model in a path diagram. Like any other method of statistical modeling, it needs conditions to work effectively.

\section{ACKNOWLEDGMENT}

The research was supported by NSF Grant DMS04-37167 and by the James McKeen Cattell Fund. We are thankful to two referees for their constructive comments that have led to a significant improvement of this article over the previous version.

\section{REFERENCES}

Anderson, J. C., \& Gerbing, D. W. (1988). Structural equation modeling in practice: A review and recommended two-step approach. Psychological Bulletin, 103, 411-423. 
Baron, R., \& Kenny, D. (1986). The moderator-mediator variable distinction in social psychological research: Conceptual, strategic, and statistical considerations. Journal of Personality and Social Psychology, 51, 1173-1182.

Bentler, P. M. (1990). Fit indexes, Lagrange multipliers, constraint changes and incomplete data in structural data. Multivariate Behavioral Research, 25, 163-172.

Bentler, P. M. (1995). EQS structural equations programmanual. Encino, CA: Multivariate Software.

Bentler, P. M., \& Chou, C.-P. (1993). Some new covariance structure model improvement statistics. In K. A. Bollen \& J. S. Long (Eds.), Testing structural equation models (pp. 235-255). Newbury Park, CA: Sage.

Boker, S. M., \& McArdle, J. J. (2005). Path analysis and path diagrams. In B. Everitt \& D. Howell (Eds.), Encyclopedia of statistics in behavioral science (Vol. 3, pp. 1529-1531). New York: Wiley.

Bollen, K. A. (1989). Structural equations with latent variables. New York: Wiley.

Bollen, K. A. (1990). A comment on model evaluation and modification. Multivariate Behavioral Research, 25, 181-185.

Bollen, K. A., \& Ting, K. (2000). A tetrad test for causal indicators. Psychological Methods, 5, $3-22$.

Chatterjee, S., Handcock, M. S., \& Simonoff, J. S. (1995). A casebook for a first course in statistics and data analysis. New York: Wiley.

Chou, C.-P., \& Bentler, P. M. (1990). Model modification in covariance structure modeling: A comparison among likelihood ratio, Lagrange multiplier, and Wald tests. Multivariate Behavioral Research, 25, 115-136.

Cole, D. A., \& Maxwell, S. E. (2003). Testing mediational models with longitudinal data: Questions and tips in the use of structural equation modeling. Journal of Abnormal Psychology, 112, 558577.

Cragg, J. G. (1968). Some effects of incorrect specification on the small-sample properties of several simultaneous-equation estimators. International Economic Review, 9, 63-86.

Farley, J. U., \& Reddy, S. K. (1987). A factorial evaluation of effects of model specification and error on parameter estimation in a structural equation model. Multivariate Behavioral Research, 22, 71-90.

Gallini, J. (1983). Misspecifications that can result in path analysis structures. Applied Psychological Measurement, 7, 125-137.

Hausman, J. A. (1978). Specification tests in econometrics. Econometrica, 46, 1251-1271.

Holzinger, K. J., \& Swineford, F. (1939). A study in factor analysis: The stability of a bi-factor solution. Chicago: University of Chicago.

Jöreskog, K. G., \& Sörbom, D. (1993). LISREL 8 user's reference guide. Chicago: Scientific Software International.

Kano, Y., Bentler, P. M., \& Mooijaart, A. (1993). Additional information and precision of estimators in multivariate structural models. In K. Matusita, M. L. Puri, \& T. Hayakawa (Eds.), Statistical sciences and data analysis (pp. 187-196). Zeist, Netherlands: VSP International Science.

Kaplan, D. (1990). Evaluating and modifying covariance structure models: A review and recommendation. Multivariate Behavioral Research, 25, 137-155.

Li, C. C. (1975). Path analysis: A primer. Pacific Grove, CA: Boxwood.

Loehlin, J. C. (2004). Latent variable models: An introduction to factor, path, and structural equation analysis (4th ed.). Mahwah, NJ: Lawrence Erlbaum Associates, Inc.

Luijben, T. C., \& Boomsma, A. (1988). Statistical guidance for model modification in covariance structure analysis. Compstat 1988, 335-340.

Luijben, T. C., Boomsma, A., \& Molenaar, I. W. (1988). Modification of factor analysis models in covariance structure analysis, a Monte Carlo study. In T. K. Dijkstra (Ed.), On model uncertainty and its statistical implications (pp. 70-101). New York: Springer-Verlag.

MacCallum, R. C., \& Austin, J. T. (2000). Applications of structural equation modeling in psychological research. Annual Review of Psychology, 51, 201-226. 
MacCallum, R. C., Roznowski, M., \& Necowitz, L. B. (1992). Model modifications in covariance structure analysis: The problem of capitalization on chance. Psychological Bulletin, 111, 490504.

Marcoulides, G. A., \& Drezner, Z. (2001). Specification searches in structural equation modeling with a genetic algorithm. In G. A. Marcoulides \& R. E. Schumacker (Eds.), New developments and techniques in structural equation modeling (pp. 247-268). Mahwah, NJ: Lawrence Erlbaum Associates, Inc.

Marcoulides, G. A., \& Drezner, Z. (2003). Model specification searches using ant colony optimization algorithms. Structural Equation Modeling, 10, 154-164.

Raykov, T., \& Penev, S. (2002). Exploring structural equation model misspecifications via latent individual residuals. In G. A. Marcoulides \& I. Moustaki (Eds.), Latent variable and latent structure models (pp. 121-134). Mahwah, NJ: Lawrence Erlbaum Associates, Inc.

Saris, W. E., Satorra, A., \& Sörbom, D. (1987). The detection and correction of specification errors in structural equation models. Sociological Methodology, 17, 105-129.

Shapiro, A., \& Browne, M. W. (1990). On the treatment of correlation structures as covariance structures. Linear Algebra and Its Applications, 127, 567-587.

Silvia, E. S. M., \& MacCallum, R. C. (1988). Some factors affecting the success of specification searches in covariance structure modeling. Multivariate Behavioral Research, 23, 297-326.

Sörbom, D. (1975). Detection of correlated errors in longitudinal data. British Journal of Mathematical and Statistical Psychology, 28, 138-151.

Sörbom, D. (1989). Model modification. Psychometrika, 54, 371-384.

Steiger, J. H. (2002). When constraints interact: A caution about reference variables, identification constraints, and scale dependencies in structural equation modeling. Psychological Methods, 7 , 210-227.

Wheaton, B., Muthén, B., Alwin, D., \& Summers, G. (1977). Assessing reliability and stability in panel models. Sociological Methodology, 8, 84-136.

Wright, S. (1920). The relative importance of heredity and environment in determining the piebald pattern of guinea-pigs. Proceedings of the National Academy of Sciences, 6, 320-332.

Yuan, K.-H., \& Bentler, P. M. (1998). Robust mean and covariance structure analysis. British Journal of Mathematical and Statistical Psychology, 51, 63-88.

Yuan, K.-H., \& Bentler, P. M. (2001). Effect of outliers on estimators and tests in covariance structure analysis. British Journal of Mathematical and Statistical Psychology, 54, 161-175.

Yuan, K.-H., \& Bentler, P. M. (2004). On chi-square difference and $z$ tests in mean and covariance structure analysis when the base model is misspecified. Educational and Psychological Measurement, 64, 737-757.

Yuan, K.-H., \& Bentler, P. M. (2007a). Robust procedures in structural equation modeling. In S.-Y. Lee \& E. J. Kontoghiorghes (Eds.), Handbook of latent variable and related models (pp. 367-397). Amsterdam: Elsevier.

Yuan, K.-H., \& Bentler, P. M. (2007b). Structural equation modeling. In C. R. Rao \& S. Sinharay (Eds.), Handbook of statistics 26: Psychometrics (pp. 297-358). Amsterdam: North-Holland.

Yuan, K.-H., \& Chan, W. (2005). On nonequivalence of several procedures of structural equation modeling. Psychometrika, 70, 791-798.

Yuan, K.-H., Chan, W., \& Bentler, P. M. (2000). Robust transformation with applications to structural equation modeling. British Journal of Mathematical and Statistical Psychology, 53, 31-50.

Yuan, K.-H., \& Hayashi, K. (2006). Standard errors in covariance structure models: Asymptotics versus bootstrap. British Journal of Mathematical and Statistical Psychology, 59, 397-417.

Yuan, K.-H., Marshall, L. L., \& Bentler, P. M. (2003). Assessing the effect of model misspecifications on parameter estimates in structural equation models. Sociological Methodology, 33, 241-265.

Yung, Y.-F., \& Bentler, P. M. (1999). On added information for ML factor analysis with mean and covariance structures. Journal of Educational and Behavioral Statistics, 24, 1-20. 


\section{APPENDIX}

This appendix provides the detail leading to Equation 2. The relation of $f_{1}, f_{2}$, and $f_{3}$ in Figure 6 are expressed in equations as

$$
\begin{gathered}
f_{2}=\gamma_{1} f_{1}+d_{2}, \\
f_{3}=\gamma_{2} f_{1}+\gamma_{3} f_{2}+d_{3} \\
=\gamma_{2} f_{1}+\gamma_{3}\left(\gamma_{1} f_{1}+d_{2}\right)+d_{3} \\
=\left(\gamma_{2}+\gamma_{1} \gamma_{3}\right) f_{1}+\gamma_{3} d_{2}+d_{3},
\end{gathered}
$$

where $E\left(f_{1}\right)=0, E\left(f_{2}\right)=0, E\left(f_{3}\right)=0, E\left(d_{2}\right)=0, E\left(d_{3}\right)=0, E\left(f_{1} d_{2}\right)=$ $0, E\left(f_{1} d_{3}\right)=0, E\left(f_{2} d_{3}\right)=0, E\left(d_{2} d_{3}\right)=0$, and $\operatorname{Var}\left(f_{1}\right)=\phi_{11}$ according to the model assumption. Thus,

$$
\begin{aligned}
\operatorname{Var}\left(f_{2}\right) & =E\left[\left(\gamma_{1} f_{1}+d_{2}\right)^{2}\right] \\
& =E\left(\gamma_{1}^{2} f_{1}^{2}+2 \gamma_{1} f_{1} d_{2}+d_{2}^{2}\right) \\
& =\gamma_{2}^{2} \phi_{11}+\varphi_{22},
\end{aligned}
$$

$$
\begin{aligned}
\operatorname{Var}\left(f_{3}\right)= & E\left\{\left[\left(\gamma_{2}+\gamma_{1} \gamma_{3}\right) f_{1}+\gamma_{3} d_{2}+d_{3}\right]^{2}\right\} \\
= & E\left[\left(\gamma_{2}+\gamma_{1} \gamma_{3}\right)^{2} f_{1}^{2}+\gamma_{3}^{2} d_{2}^{2}+d_{3}^{2}+2\left(\gamma_{2}+\gamma_{1} \gamma_{3}\right) \gamma_{3} f_{1} d_{2}\right. \\
& \left.+2\left(\gamma_{2}+\gamma_{1} \gamma_{3}\right) f_{1} d_{3}+2 \gamma_{3} d_{2} d_{3}\right] \\
= & \left(\gamma_{2}+\gamma_{1} \gamma_{3}\right)^{2} \phi_{11}+\gamma_{3}^{2} \varphi_{22}+\varphi_{33},
\end{aligned}
$$

$$
\begin{aligned}
\operatorname{Cov}\left(f_{1}, f_{2}\right) & =E\left[f_{1}\left(\gamma_{1} f_{1}+d_{2}\right)\right] \\
& =\gamma_{1} \phi_{11},
\end{aligned}
$$

$$
\begin{aligned}
\operatorname{Cov}\left(f_{1}, f_{3}\right) & =E\left\{f_{1}\left[\left(\gamma_{2}+\gamma_{1} \gamma_{3}\right) f_{1}+\gamma_{3} d_{2}+d_{3}\right]\right\} \\
& =\left(\gamma_{2}+\gamma_{1} \gamma_{3}\right) \phi_{11},
\end{aligned}
$$

$$
\begin{aligned}
\operatorname{Cov}\left(f_{2}, f_{3}\right)= & E\left\{\left(\gamma_{1} f_{1}+d_{2}\right)\left[\left(\gamma_{2}+\gamma_{1} \gamma_{3}\right) f_{1}+\gamma_{3} d_{2}+d_{3}\right]\right\} \\
= & E\left[\gamma_{1}\left(\gamma_{2}+\gamma_{1} \gamma_{3}\right) f_{1}^{2}+\gamma_{1} \gamma_{3} f_{1} d_{2}+\gamma_{1} f_{1} d_{3}\right. \\
& \left.+\left(\gamma_{2}+\gamma_{1} \gamma_{3}\right) f_{1} d_{2}+\gamma_{3} d_{2}^{2}+d_{2} d_{3}\right] \\
= & \gamma_{1}\left(\gamma_{2}+\gamma_{1} \gamma_{3}\right) \phi_{11}+\gamma_{3} \varphi_{33} .
\end{aligned}
$$

Putting these elements into a matrix yields Equation 2. 